\title{
Molecular signatures and phylogenomic analysis of the genus Burkholderia: proposal for division of this genus into the emended genus Burkholderia containing pathogenic organisms and a new genus Paraburkholderia gen. nov. harboring environmental species
}

\author{
Amandeep Sawana, Mobolaji Adeolu and Radhey S. Gupta * \\ Department of Biochemistry and Biomedical Sciences, Health Sciences Center, McMaster University, Hamilton, ON, Canada
}

\section{Edited by:}

Scott Norman Peterson, Sanford

Burnham Medical Research

Institute, USA

\section{Reviewed by:}

Loren John Hauser, Oak Ridge National Laboratory, USA

William Charles Nierman, J. Craig

Venter Institute, USA

\section{${ }^{*}$ Correspondence:}

Radhey S. Gupta, Department of Biochemistry and Biomedical Sciences, Health Sciences Center, McMaster University, 1200 Main Street West, Hamilton, ON L8N 3Z5, Canada

e-mail: gupta@mcmaster.ca
The genus Burkholderia contains large number of diverse species which include many clinically important organisms, phytopathogens, as well as environmental species. However, currently, there is a paucity of biochemical or molecular characteristics which can reliably distinguish different groups of Burkholderia species. We report here the results of detailed phylogenetic and comparative genomic analyses of 45 sequenced species of the genus Burkholderia. In phylogenetic trees based upon concatenated sequences for 21 conserved proteins as well as 16S rRNA gene sequence based trees, members of the genus Burkholderia grouped into two major clades. Within these main clades a number of smaller clades including those corresponding to the clinically important Burkholderia cepacia complex (BCC) and the Burkholderia pseudomallei groups were also clearly distinguished. Our comparative analysis of protein sequences from Burkholderia spp. has identified 42 highly specific molecular markers in the form of conserved sequence indels (CSIs) that are uniquely found in a number of well-defined groups of Burkholderia spp. Six of these CSIs are specific for a group of Burkholderia spp. (referred to as Clade I in this work) which contains all clinically relevant members of the genus (viz. the BCC and the B. pseudomallei group) as well as the phytopathogenic Burkholderia spp. The second main clade (Clade II), which is composed of environmental Burkholderia species, is also distinguished by 2 identified CSIs that are specific for this group. Additionally, our work has also identified multiple CSIs that serve to clearly demarcate a number of smaller groups of Burkholderia spp. including 3 CSIs that are specific for the B. cepacia complex, 4 CSIs that are uniquely found in the B. pseudomallei group, 5 CSIs that are specific for the phytopathogenic Burkholderia spp. and 22 other CSI that distinguish two groups within Clade II. The described molecular markers provide highly specific means for the demarcation of different groups of Burkholderia spp. and they also offer novel and useful targets for the development of diagnostic assays for the clinically important members of the BCC or the pseudomallei groups. Based upon the results of phylogenetic analyses, the identified CSIs and the pathogenicity profile of Burkholderia species, we are proposing a division of the genus Burkholderia into two genera. In this new proposal, the emended genus Burkholderia will correspond to the Clade I and it will contain only the clinically relevant and phytopathogenic Burkholderia species. All other Burkholderia spp., which are primarily environmental, will be transferred to a new genus Paraburkholderia gen. nov.

Keywords: Burkholderia, Burkholderia cepacia complex, conserved signature indels, phylogenetic trees, molecular signatures

\section{INTRODUCTION}

The genus Burkholderia is a morphologically, metabolically, and ecologically diverse group of gram-negative bacteria (Yabuuchi et al., 1992; Coenye and Vandamme, 2003; Mahenthiralingam et al., 2005; Palleroni, 2005; Compant et al., 2008). Burkholderia species are ubiquitous in the environment (Coenye and
Vandamme, 2003). They inhabit a wide range of ecological niches, ranging from soil to the human respiratory tract (Coenye and Vandamme, 2003). A group of 17 closely related Burkholderia species, the Burkholderia cepacia complex (BCC), are responsible for prevalent and potentially lethal pulmonary infections in immunocompromised individuals, such as individuals with cystic 
fibrosis (Mahenthiralingam et al., 2002, 2005; Biddick et al., 2003; Hauser et al., 2011). Burkholderia pseudomallei, a Burkholderia species related to the BCC, is the causative agent for the disease melioidosis, a potentially lethal septic infection which accounts for up to $20 \%$ of all community-acquired septicemias in some regions (White, 2003; Limmathurotsakul and Peacock, 2011). Other species related to the BCC are the causative agents of major infections in both animals (Burkholderia mallei) and plants (Burkholderia glumae and Burkholderia gladioli) (Whitlock et al., 2007; Nandakumar et al., 2009).

In spite of the large diversity and varied pathogenicity among the $>70$ members of the group, all Burkholderia species are currently placed within one genus (Coenye and Vandamme, 2003; Palleroni, 2005). The phylogeny and taxonomy of the genus Burkholderia is primarily defined on the basis of $16 \mathrm{~S}$ rRNA sequence analysis (Yabuuchi et al., 1992; Palleroni, 2005; Yarza et al., 2008). The inferences obtained from $16 \mathrm{~S}$ rRNA analysis have been further substantiated by other phylogenetic methods, including recA gene based analysis (Payne et al., 2005), acdS gene based analysis (Onofre-Lemus et al., 2009), DNA-DNA hybridization (Gillis et al., 1995), whole cell fatty acid analysis (Stead, 1992), multilocus sequence analysis (Tayeb et al., 2008; Spilker et al., 2009; Estrada-de los Santos et al., 2013), gene gain/loss analysis (Zhu et al., 2011), and whole genome phylogenetic analysis (Ussery et al., 2009; Segata et al., 2013). In many of these phylogenetic studies, the members of the genus Burkholderia can be divided into two or more distinct phylogenetic groups, with one group consisting of members of the BCC and related species (Payne et al., 2005; Tayeb et al., 2008; Yarza et al., 2008; Spilker et al., 2009; Ussery et al., 2009; Gyaneshwar et al., 2011; Vandamme and Dawyndt, 2011; Zhu et al., 2011; Estrada-de los Santos et al., 2013; Segata et al., 2013). Although there are some commonly shared features among closely related groups of Burkholderia species, there is no known morphological, biochemical, or molecular characteristic specific to the larger phylogenetic groups within the genus (ex. the BCC and related species).

The advent of next generation sequencing methods has led to a rapid increase in the number of genome sequences available for bacterial species (Mardis, 2008). The availability of these sequences for members of the genus Burkholderia provides us better means to evaluate the phylogenetic relationships among different species (Ciccarelli et al., 2006; Wu et al., 2009). Importantly, the large data sets of sequences allows for the use of comparative genomic techniques to discover novel molecular markers that can provide independent evidence for different phylogenetic groups within the genus Burkholderia (Gupta, 1998, 2014; Gao and Gupta, 2012). In this work, we describe one type of molecular marker, conserved sequence insertions or deletions (CSIs), which are uniquely present in protein sequences from a defined group of organisms, that can be used to delineate different phylogenetic groups of Burkholderia species independently of traditional phylogenetic methods (Gupta, 1998, 2001; Gao and Gupta, 2012). Our comparative analysis of Burkholderia genomes has led to the identification of 42 unique CSIs that delineate different phylogenetic groups within the genus in clear molecular terms. A clade of Burkholderia containing the BCC and related organisms (Clade
I) was supported by both phylogenetic evidence and 6 identified CSIs. We have also identified 3 CSIs specific for the BCC, 4 CSIs specific for the B. pseudomallei group, and 5 CSIs specific for the plant pathogenic Burkholderia spp. The remaining members of the genus Burkholderia formed another monophyletic clade (Clade II) in our phylogenetic trees which was supported by 2 CSIs. Within Clade II, we identified two smaller clades of Burkholderia that were supported by 16 and 6 CSIs. The grouping of members of the genus Burkholderia into at least two large, monophyletic groups has also been observed in a large body of prior phylogenetic research (Payne et al., 2005; Tayeb et al., 2008; Yarza et al., 2008; Spilker et al., 2009; Ussery et al., 2009; Gyaneshwar et al., 2011; Zhu et al., 2011; Estrada-de los Santos et al., 2013; Segata et al., 2013). Based on the phylogenetic evidence and our identified CSIs, we propose division of the genus Burkholderia into two genera: an emended genus Burkholderia containing clinically important and phytopathogenic members of the genus and a new genus Paraburkholderia gen. nov. harboring the environmental species.

\section{MATERIALS AND METHODS PHYLOGENETIC ANALYSIS}

A concatenated sequence alignment of 21 highly conserved proteins (viz. ArgRS, EF-G, GyrA, GyrB, Hsp60, Hsp70, IleRS, RecA, RpoB, RpoC, SecY, ThrRS, TrpS, UvrD, ValRS, 50S ribosomal proteins L1, L5 and L6, and 30S ribosomal proteins S2, S8 and S11) was used to perform phylogenetic analysis. Due to their presence in most bacteria, these proteins have been extensively utilized for phylogenetic studies (Gupta, 1998, 2009; Kyrpides et al., 1999; Harris et al., 2003; Charlebois and Doolittle, 2004; Ciccarelli et al., 2006). The amino acid sequences for these conserved proteins were obtained from NCBI database for all of the species/strains listed in Table 1, which includes 45 sequenced species of the genus Burkholderia. Furthermore, three genomes from other members of class Betaproteobacteria (viz. Cupriavidus necator N-1, Bordetella pertussis Tohama I, and Neisseria meningitides MC58), serving as outgroups in our analysis, were also retrieved from NCBI database. Depending on genome availability, type strains were selected for most of the species. Multiple sequence alignments for these proteins were created using Clustal_X 1.83 and concatenated into a single alignment file (Jeanmougin et al., 1998). Poorly aligned regions from the alignment file were removed using Gblocks $0.91 \mathrm{~b}$ and the resulting alignment, which contained 7688 aligned characters, was ultimately utilized for phylogenetic analysis (Castresana, 2000). A maximum likelihood (ML) tree based on 100 bootstrap replicates of this alignment was constructed using MEGA 6.0 while employing Jones-TaylorThornton substitution model (Jones et al., 1992; Tamura et al., 2013).

A maximum likelihood 16S rRNA gene sequence consensus tree was also created for 101 sequences, which included 97 representative strains from the genus Burkholderia and four outgroup sequences from the genera Cupriadivus and Ralstonia. The sequences utilized in the study were obtained from the Ribosomal Database Project (RDP III) (Cole et al., 2009) and NCBI. All the sequences were aligned using MAAFT 7 (Katoh and Standley, 2013) and a ML tree based upon 1000 bootstrap replicates of 
Table 1 | Genome characteristics of the sequenced members of the genus Burkholderia.

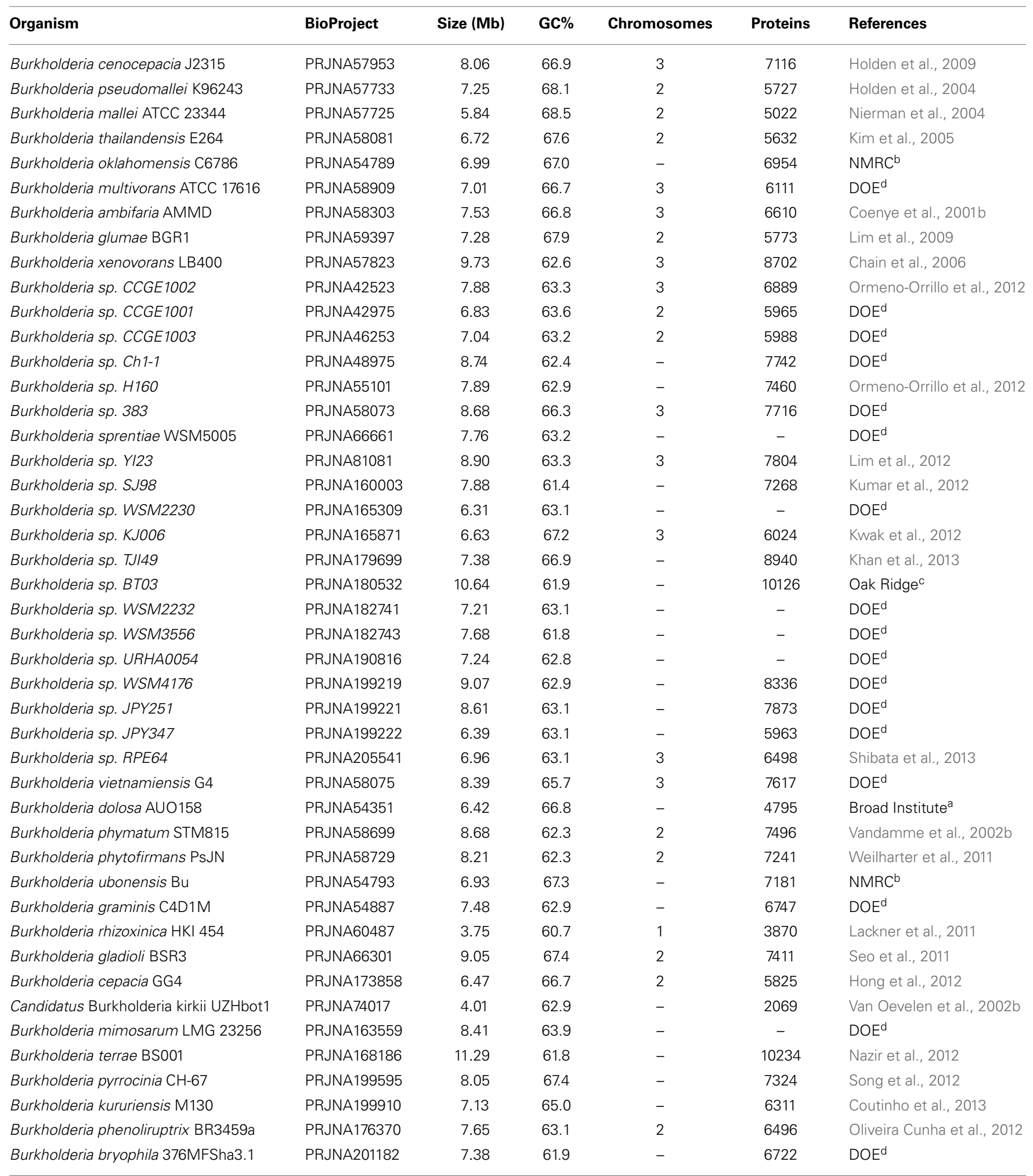

${ }^{a}$ The Broad Institute Genome Sequencing Platform (Broad Institute).

${ }^{b}$ Naval Medical Research Center/ Biological Defense Research Directorate (NMRC).

c Oak Ridge National Lab (Oak Ridge).

${ }^{d} D O E$ Joint Genome Institute (DOE). 
this alignment was constructed using the General Time Reversible Model (Tavaré, 1986) in MEGA 6.0 (Tamura et al., 2013).

\section{IDENTIFICATION OF MOLECULAR MARKERS (CSIs)}

BLASTp searches were conducted for all proteins from chromosomes 2 and 3 (accession numbers NC_008061 and NC_008061) of Burkholderia cenocepacia J2315 (Holden et al., 2009) to identify CSIs that are shared by different members of the genus Burkholderia. Species that appeared as top hits with high scoring homologs $\left(E\right.$ values $\left.<1 \mathrm{e}^{-20}\right)$ from the genus Burkholderia and other outgroups were selected. Multiple sequence alignments were created using the Clustal_X 1.83 (Jeanmougin et al., 1998). These alignments were visually inspected for the presence of insertions or deletions (indels) restricted to either some or all members of the genus Burkholderia and flanked by at least 5-6 conserved amino acid residues on both sides in the neighboring 30-40 amino acids. Indel queries that were not flanked by conserved regions were not further evaluated. The species specificity of the indel queries meeting the above criterion was further evaluated by performing BLASTp searches on short sequence segments containing the insertions or deletions, and their flanking conserved regions (60-100 amino acids long). The searches were conducted against the NCBI non-redundant (nr) database and a minimum of 250 BLAST hits were examined for the presence or absence of CSIs. The results of these analyses were evaluated as described in detail in our recent work (Gupta, 2014). Signature files for the CSIs that were specific for members of the genus Burkholderia were created and formatted using the programs SIG_CREATE and SIG_STYLE (accessible from Gleans.net) as described by Gupta (2014). The sequence alignment files presented here contain information for all detected insertions or deletions from the Burkholderia group of interest, but only a limited number from species that are serving as outgroups. Sequence information for different strains of various species is not shown, but they all exhibited similar pattern. Lastly, unless otherwise indicated, the CSIs shown here are specifically found in the indicated groups and similar CSIs were not detected in the 250 Blast hits with the query sequences.

\section{RESULTS}

\section{BRANCHING PATTERN OF BURKHOLDERIA SPECIES IN CONCATENATED PROTEIN AND 16S rRNA TREES}

Genome sequences of 45 species of Burkholderia were available from the NCBI genome database at the time of this work (NCBI, 2014). Some characteristics of these genomes are listed in Table 1. The genome sizes of the sequenced Burkholderia species show large variation (from 3.75-11.29 Mb) and the numbers of proteins in them also varied in a similar proportion. In this work we have produced a ML phylogenetic tree based on the concatenated amino acid sequences of 21 conserved housekeeping and ribosomal proteins obtained from 45 sequenced Burkholderia species (Figure 1). The Burkholderia species formed two large clades in the protein based ML tree: One consisting of the BCC and related organisms (Clade I) and another comprised mainly of environmental or poorly characterized Burkholderia species (Clade II). Within Clade I, three smaller, distinct clades are also observed. The first of these clades (Clade Ia) is wholly comprised of the sequenced BCC species, the second clade (Clade $\mathrm{Ib}$ ) groups B. pseudomallei and closely related species, and the third clade (Clade Ic) consists of the plant pathogenic species, B. glumae and B. gladioli. Clade II could also be divided into two smaller clades, Clade IIa and Clade IIb. Clade IIa is separated from Clade IIb by a long branch, suggesting that a large amount of genetic divergence has occurred between the two groups. In addition to the two main clades of Burkholderia, two species, Burkholderia sp. JPY347 and Burkholderia rhizoxinica, branched early in the tree and did not associate with either Clade I or II.

We have also constructed a $16 \mathrm{~S}$ rRNA based ML phylogenetic tree for 97 Burkholderia strains and candidate species (Figure 2). In this 16S rRNA based phylogenetic tree we observed broadly similar patterns to our protein based phylogeny. A clade consisting of the BCC and related organisms (Clade I) was clearly resolved. The three subclades within Clade I, the BCC (Clade Ia), the B. pseudomallei group (Clade Ib), and the plant pathogenic species (Clade Ic) were well resolved, though some species exhibited aberrant branching (ex. B. oklahomensis and B. pseudomultivorans). A large assemblage of the remaining Burkholderia species, roughly corresponding to Clade II in our concatenated protein based phylogenetic tree, was also observed in the 16S rRNA tree. However, due to significant number of unsequenced Burkholderia species which are present in the 16S rRNA database it is difficult to accurately identify the groups within Clade II of the 16S rRNA tree which correspond to Clades IIa and IIb in our concatenated protein based phylogenetic tree. Bootstrap support for branches in the $16 \mathrm{~S}$ rRNA based tree were also significantly lower than they were in the concatenated protein tree indicating that some of the observed branching patterns may not be reliable. However, the clade consisting of the BCC and related organisms (Clade I) has strong bootstrap support and has been identified in a large number of previous 16S rRNA based phylogenetic studies (Yabuuchi et al., 1992; Palleroni, 2005; Yarza et al., 2008; Suarez-Moreno et al., 2012).

\section{MOLECULAR SIGNATURES DISTINGUISHING THE CLADE I AND CLADE II BURKHOLDERIA}

Rare genetic changes, such as insertions and deletions in essential genes/proteins, which occur in a common ancestor can be inherited by the various decedent species related to this common ancestor (Gupta, 1998; Rokas and Holland, 2000; Gogarten et al., 2002; Gupta and Griffiths, 2002). Due to the rarity and the specific presence of these rare genetic changes to a related group of organisms, they can serve as important molecular markers and provide a novel means to understand the evolutionary interrelationships between different closely related species (Gupta, 1998; Gupta and Griffiths, 2002; Gao and Gupta, 2012).

The comparative analysis of protein sequences from Burkholderia species that was carried out in the present work has identified a number of CSIs that serve to clearly distinguish a number of different clades within the genus Burkholderia. These studies have led to identification of 6 CSIs that are specific for the Clade I Burkholderia, consisting of the BCC and related organisms, enabling clear distinction of this group from all other Burkholderia. This clade, which contains all well characterized pathogens within the genus, represents the most clinically 


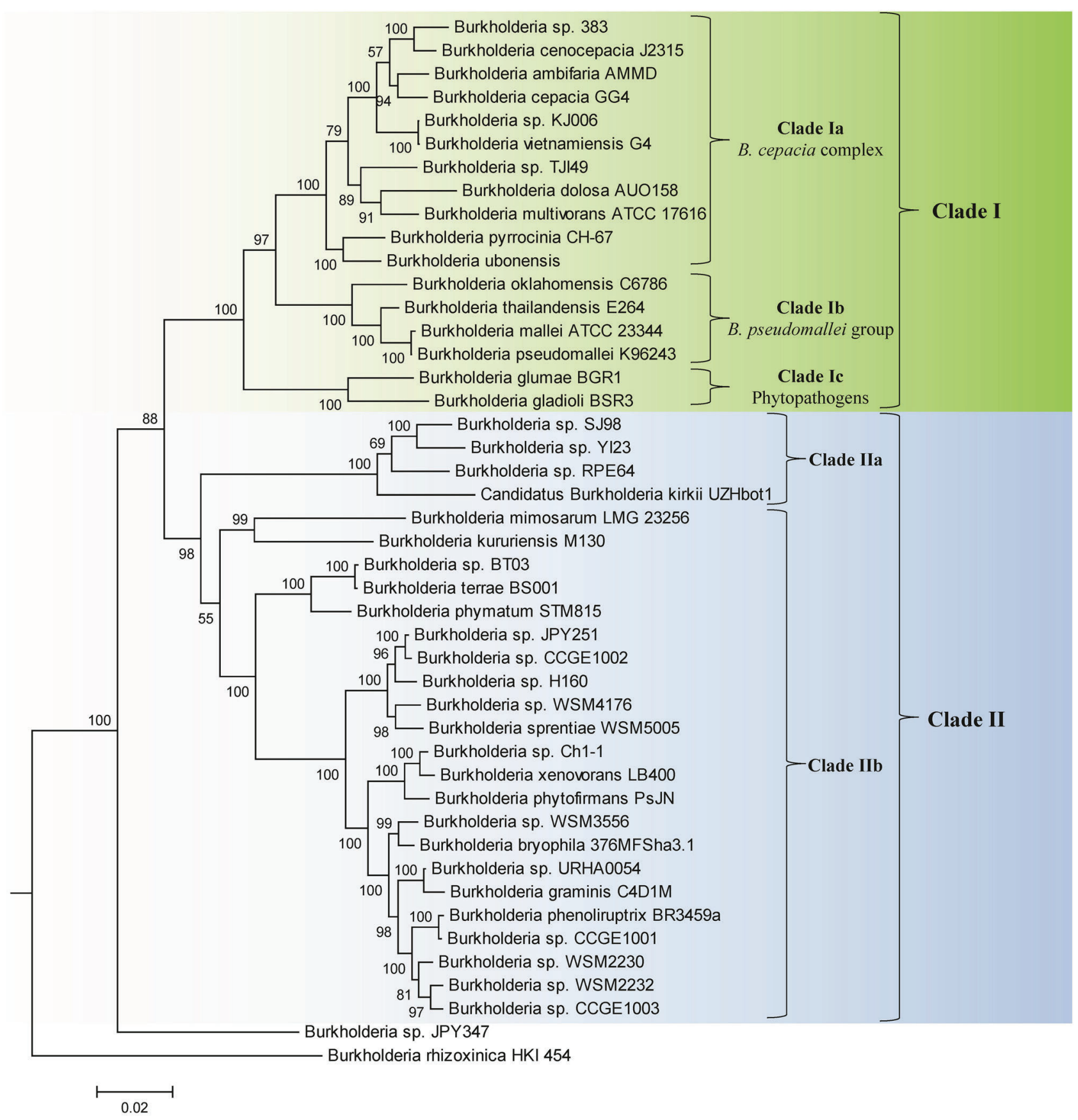

FIGURE 1|A maximum likelihood phylogenetic tree of the genome sequenced members of the genus Burkholderia based upon concatenated sequences of 21 conserved proteins. The tree was rooted using Cupriavidus necator $\mathrm{N}-1$, Bordetella pertussis
Tohama I, and Neisseria meningitides MC58. Bootstrap analysis scores are indicated for each node. The major Burkholderia clades (Clades I and II) and their main sub-clades are indicated by brackets. relevant group within the Burkholderia. All species within this clade are potentially pathogenic to human, animals, or plants and most have been isolated from clinical human samples (Simpson et al., 1994; Mahenthiralingam et al., 2002, 2005; Biddick et al., 2003; O'Carroll et al., 2003). One example of a CSI that is specific to the Clade I Burkholderia is shown in Figure 3A. In this case, a one amino acid deletion is present in a highly conserved region of a periplasmic amino acid-binding protein. The indel is flanked on both sides by highly conserved regions indicating that it is not the result of alignment artifacts and that it is a reliable genetic characteristic. This CSI is present in all of the sequenced members of the Clade I Burkholderia, but absent in all other bacterial homologs of this protein. Our work has identified 5 additional CSIs in other widely distributed proteins that are 


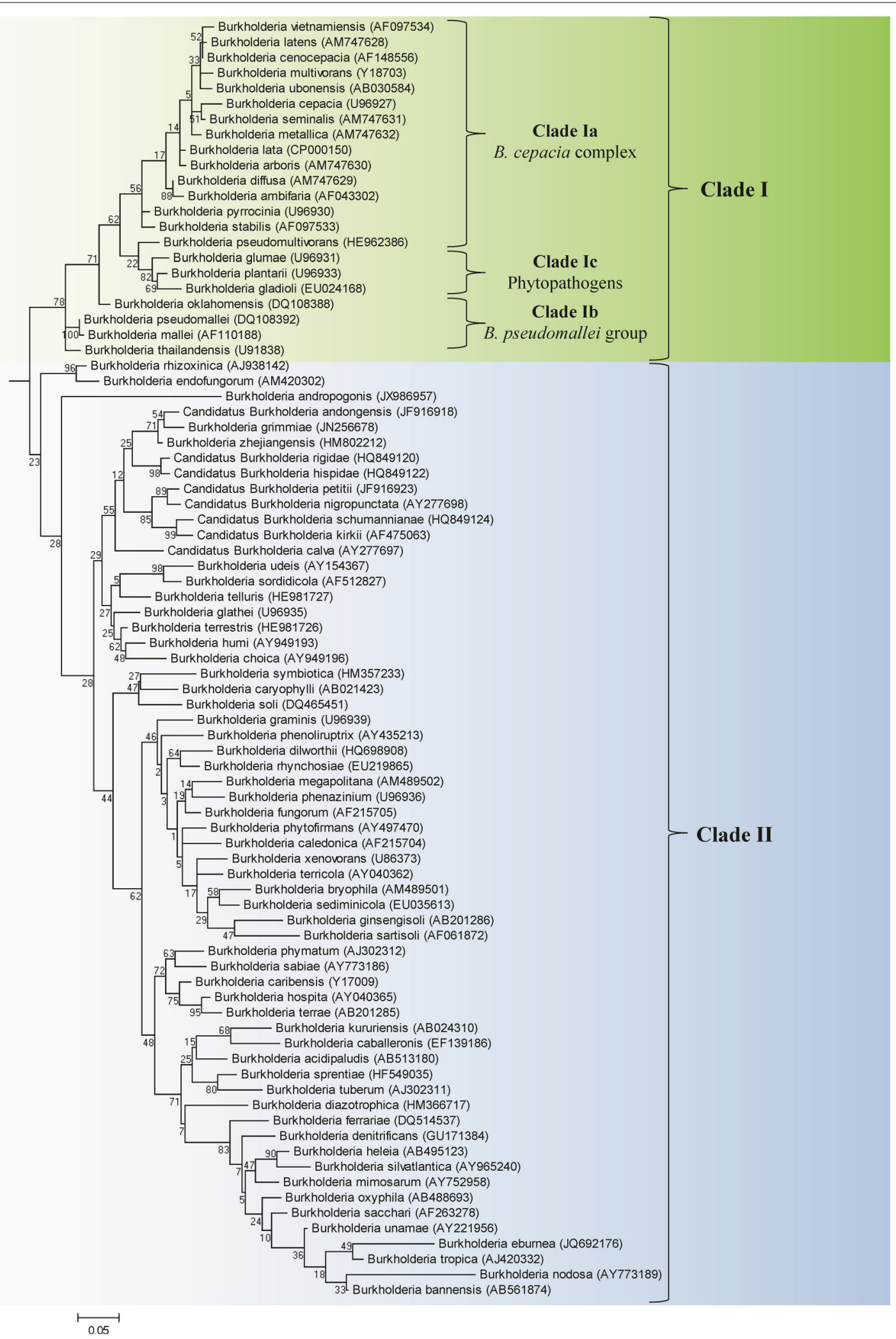

FIGURE 2 | A maximum likelihood tree based on the 16S rRNA gene sequences of $\mathbf{9 7}$ members of the genus Burkholderia. Accession numbers for the 16S rRNA sequenced used for each organism are provided in the brackets following the name of the organism. The tree was rooted using four species from the genera Cupriadivus and Ralstonia. Bootstrap analysis scores are indicated for each node. The major Burkholderia clades (Clades I and II) and the subclades within Clade I are indicated by brackets. 
A

\begin{tabular}{|c|c|}
\hline $\begin{array}{c}\text { Clade I } \\
\text { Burkholderia }\end{array}$ & $\begin{array}{l}\text { Burkholderia pseudomallei } 1258 \\
\text { Burkholderia thailandensis MSM } \\
\text { Burkholderia mallei GB8 horse } \\
\text { Burkholderia oklahomensis C678 } \\
\text { Burkholderia multivorans ATCC } \\
\text { Burkholderia sp. TJI49 } \\
\text { Burkholderia sp. } 383 \\
\text { Burkholderia ambifaria AMMD } \\
\text { Burkholderia vietnamiensis G4 } \\
\text { Burkholderia cenocepacia MC0-3 } \\
\text { Burkholderia sp. KJ006 } \\
\text { Burkholderia cepacia GG4 } \\
\text { Burkholderia dolosa AU0158 } \\
\text { Burkholderia glumae BGR1 } \\
\text { Burkholderia ubonensis Bu } \\
\text { Burkholderia gladioli BSR3 } \\
\text { Burkholderia pyrrocinia }\end{array}$ \\
\hline $\begin{array}{c}\text { Other } \\
\text { Bacteria }\end{array}$ & $\begin{array}{l}\text { Burkholderia sp. CCGE1002 } \\
\text { Burkholderia sp. H160 } \\
\text { Burkholderia sp. SJ98 } \\
\text { Burkholderia sp. YI23 } \\
\text { Burkholderia terrae BS001 } \\
\text { Candidatus Burkholderia kirkii } \\
\text { Burkholderia phymatum STM815 } \\
\text { Burkholderia phytofirmans PSJN } \\
\text { Burkholderia graminis C4D1M } \\
\text { Burkholderia sp. CCGE1001 } \\
\text { Burkholderia sp. CCGE1003 } \\
\text { Burkholderia sp. Ch1-1 } \\
\text { Burkholderia xenovorans LB400 } \\
\text { Burkholderia rhizoxinica HKI } 4 \\
\text { Burkholderia cepacia GG4 } \\
\text { Burkholderia sp. BT03 } \\
\text { - Burkholderia phenoliruptrix BR } \\
\text { Ralstonia solanacearum IP01609 } \\
\text { blood disease bacterium R229 } \\
\text { - Cupriavidus basilensis OR16 }\end{array}$ \\
\hline
\end{tabular}

135

385357135 PAYTQQGFKTTYRVVATDAQQGPALANYARS

167838282

67643044

167571630

161523366

325526658

78064858

115350248

134294354

170731615

387900963

402564996

254250888

238025878

167587824

330815169

515904347

295675201

209519165

384923770

377822189

390569671

35054392

186474882

187922400

170693943

323524494

307728219

385207261

91781519

312797511

402570122

398043025

407714518

207742583

344170252

374371980

PAYTQG

-

-

-

-

-

-

-

-

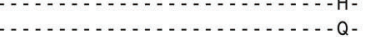

-

-

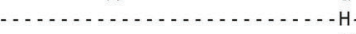

- $\begin{array}{ll} & -1 \\ N\end{array}$

-1
-1

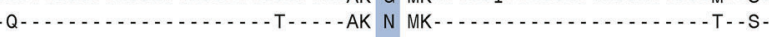

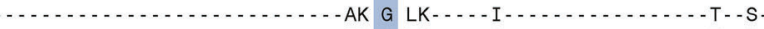

-

..............................

.

.

.

-

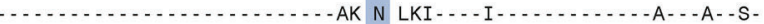

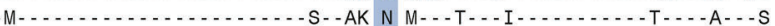

.

.

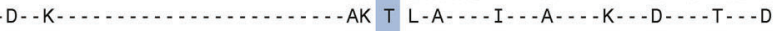

D- K- .

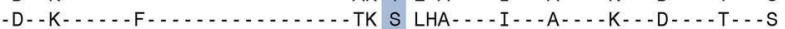

B

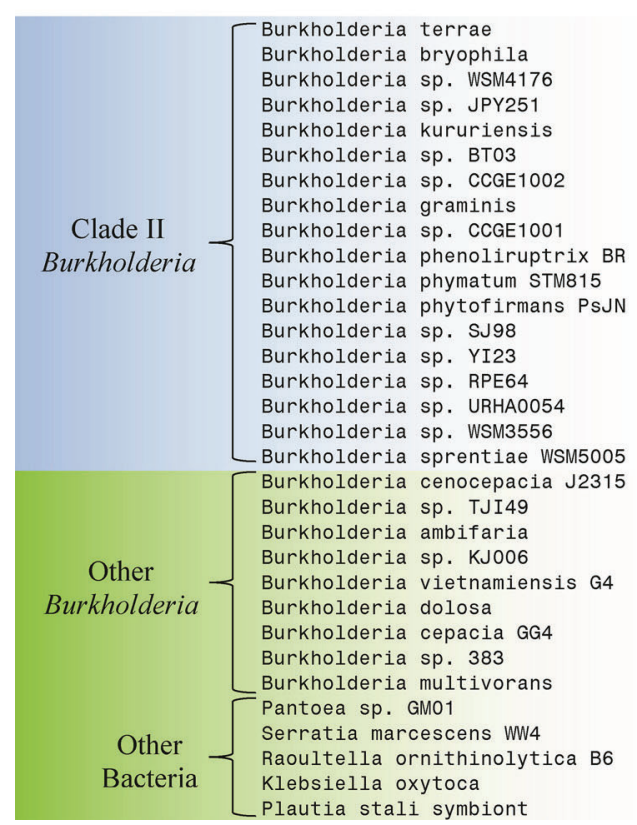

497456569

518912087

517236495

517249394

516385959

495017440

295699666

492923470

323528076

407708911

186474061

187920153

495628822

377812899

507526324

522812221

548691604

548699442

206563985

497376604

493809762

387905792

134294110

493818725

402568272

78061633

493455325

495165920

448241808

481848689

490204383

498301474
279

DPAAFDAPNAEAEADAFVEWVKASPLAGG A ERIYAPGEPERATRAEREANGIPVD

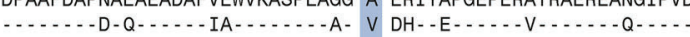

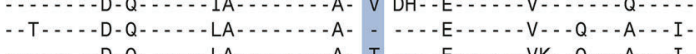

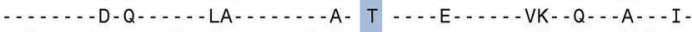

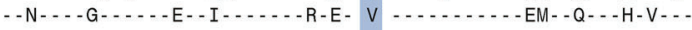

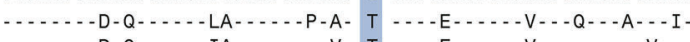

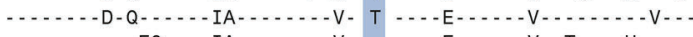
-

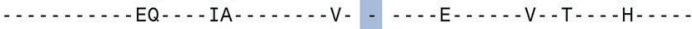
- D.

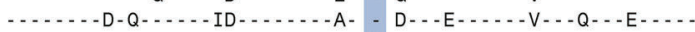

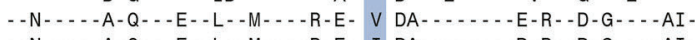

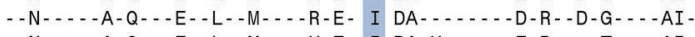

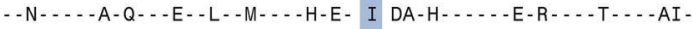

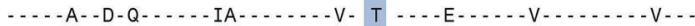

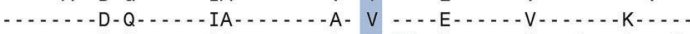

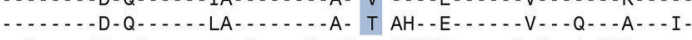

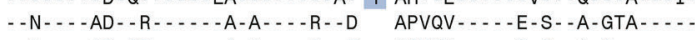

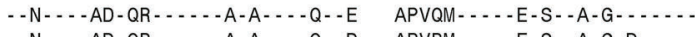

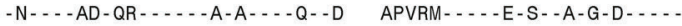

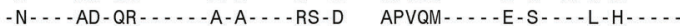

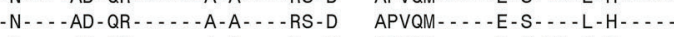
-N - - AD-QR -...A-A-..Q-D APVQM-..-Q-S-VA-T-E-...

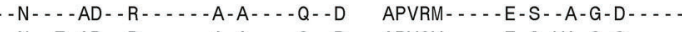

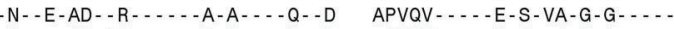

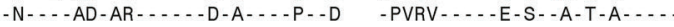
N-D - . - AMQE - - ES-LD - - Q- - QS - D AP-QV - - W-EQN - VT-GRD - - L -Q - . - QMQ - - E- - IG - ...-PS-E QP-AV - -W-E-N--A-LEQ - - I -K- - - - DMQR-TE - ID-C-Q- - HDAQ TP-LV - - W-E-N-QA-L-Q-.-L S-E - . - - DMQR-TE- - ID-C-Q- -HEPD AP-L-..-W-E-N-QQ-L-E-.-L N-D - . . - AMQR - - EE-LA - RR - - QS-E AE-LV - -W-TRN- - A - RE - - L -
FIGURE 3 | Partial sequence alignments of (A) a periplasmic amino acid-binding protein showing a 1 amino acid deletion identified in all members of Clade I of the genus Burkholderia (B) a dehydrogenase showing a 1 amino acid insertion (boxed) identified only in members of Clade II of the genus Burkholderia. These CSIs were not found in the sequence homologs of these proteins from any other sequenced bacteria. In each case, sequence information for a Burkholderia species and a limited number other bacteria are shown, but unless otherwise indicated, similar
CSIs were detected in all members of the indicated group and not detected in any other bacterial species in the top 250 BLAST hits. The dashes $(-)$ in the alignments indicate identity with the residue in the top sequence. GenBank identification (GI) numbers for each sequence are indicated in the second column. Sequence information for other CSIs specific to the members of Clade I and Clade II of the genus Burkholderia are presented in Supplemental Figures $1-5$ and Supplemental Figure 6 , respectively, and their characteristics are summarized in Table $\mathbf{2}$. 
specific for the Clade I Burkholderia and sequence alignments for these CSIs are shown in Supplemental Figures 1-5 and a summary of their characteristics is provided in Table 2.

Two additional CSIs identified in this work are specific for the Clade II Burkholderia species which is made up of mainly environmental organisms. One of these CSIs, shown in Figure 3B, consists of a one amino acid insertion in a dehydrogenase protein that is uniquely found in members of the Clade II Burkholderia and absent in all other Burkholderia species as well all other bacterial groups. A sequence alignment for another CSI that is specific for the Clade II Burkholderia (a 2 aa deletion in a LysR family of transcription regulator protein) is shown in Supplemental Figure 6 and its characteristics are summarized in Table 2 .

\section{CSIS DISTINGUISHING DIFFERENT MAIN GROUPS WITHIN THE CLADE I BURKHOLDERIA}

The species within Clade I of the genus Burkholderia are responsible for a range of human, animal, and plant diseases (Biddick et al., 2003; Mahenthiralingam et al., 2005). The members of Clade I (i.e., the BCC and related Burkholderia) are commonly separated into 3 main groups which correspond to clades identified in our phylogenetic trees. The first group, the members of the BCC (Clade 1a), are prevalent pathogens in cystic fibrosis patients, the second group, the $B$. pseudomallei group (Clade $\mathrm{Ib}$ ), contains the causative agents of melioidosis and glanders, while the third group contains the plant pathogenic Burkholderia species (Clade Ic) (White, 2003; Mahenthiralingam et al., 2005; Whitlock et al., 2007; Nandakumar et al., 2009). Our analysis has identified 3 CSIs that are specific for all members of the BCC clade (Clade 1a). One example of a BCC clade specific CSI is shown in Figure 4A. This CSI consists of a 2 amino acid insertion in a conserved region of a histidine utilization repressor which is only found in members of the BCC. Sequence alignments for two other BCC clade specific CSIs are shown in Supplemental Figures 7, 8 and their characteristics are summarized in Table 3.

Our work has also identified 4 CSIs that are specific for the $B$. pseudomallei group (Clade $\mathrm{Ib}$ ) which contains the most prevalent human pathogen within the genus, $B$. pseudomalle $i$ (Wiersinga et al., 2006). One example of a CSI specific to the B. pseudomallei group, which consists of a 1 amino acid insertion in a conserved region of a periplasmic oligopeptide-binding protein, is shown in Figure 4B. Sequence alignments for three other CSIs in three different proteins that are specific for the B. pseudomallei group are shown in Supplemental Figures 9-11 and their characteristics are summarized in Table 3.

We have also identified 5 CSIs that are specific for the major plant pathogenic group within the genus Burkholderia (Clade 1c) which contains the species B. glumae and B. gladioli. An example of a CSI representing this group is shown in Figure 4C. This CSI consists of a 1 amino acid insertion in a conserved region of a SMP-30/gluconolaconase/LRE-like region-containing protein that is found in the members of Clade $1 \mathrm{c}$ of the genus Burkholderia but absent in all other Burkholderia and all other bacterial groups. Sequence alignments for the other 4 CSIs are shown in Supplemental Figures 12-15 and their key features are highlighted in Table 3.

\section{CSIS THAT ARE SPECIFIC FOR TWO GROUPS WITHIN THE CLADE II BURKHOLDERIA}

The species within Clade II of the genus Burkholderia inhabit a variety of environmental niches, but there is little evidence of their colonization of healthy or immunocompromised human patients (Coenye and Vandamme, 2003). The branching of different groups within Clade II is not well resolved in $16 \mathrm{~S}$ rRNA trees and there is currently a lack of sequence data that can be used to generate trees based on concatenated gene sets that reliably resolve the interrelationships of the clade while sufficiently reflecting the total diversity of species within the clade (Figures 1, 2) (Cole et al., 2009; NCBI, 2014). Despite the limited sequence data, we have been able to identify two robust groups within Clade II that are supported by a number of CSIs. The first Clade, Clade IIa, primarily consists of unclassified members of the genus and candidatus Burkholderia species (Figure 1). Clade IIa is supported by 16 CSIs identified in this work. One example of a CSI specific for Clade IIa, consisting of a 1 amino acid insertion in 3-phosphoglycerate dehydrogenase, is shown in Figure 5A. This insertion is present in a highly conserved region of this protein in all sequenced members of Clade IIa and absent in all other Burkholderia and all other bacterial groups. Sequence alignments for the other 15 CSIs that are specific for Clade IIa Burkholderia spp. are shown in Supplemental Figures 16-30 and their characteristics are summarized in Table 3.

Table 2 | Conserved signature indels specific for the two major clades within the genus Burkholderia.

\begin{tabular}{|c|c|c|c|c|c|}
\hline Protein Name & GI Number & Figures & Indel size & Indel position ${ }^{a}$ & Specificity \\
\hline Periplasmic amino acid-binding protein & 385357135 & Figure 3A & 1 aa del & $135-195$ & Clade I \\
\hline Putative lyase & 167724527 & Supplemental Figure 1 & 1 aa del & $70-121$ & Clade I \\
\hline 4-hydroxybenzoate 3-monooxygenase & 238023559 & Supplemental Figure 2 & 1 aa ins & $101-171$ & Clade I \\
\hline 6-phosphogluconate dehydrogenase, decarboxylating & 330820932 & Supplemental Figure 3 & 1 aa ins & $137-202$ & Clade I \\
\hline Putative lipoprotein & 121598811 & Supplemental Figure 4 & 1 aa del & 363-393 & Clade I \\
\hline Sarcosine oxidase subunit alpha & 493818877 & Supplemental Figure 5 & 3 aa ins & $904-965$ & Clade I \\
\hline Dehydrogenase & 497456569 & Figure 3B & 1 aa ins & $279-333$ & Clade II \\
\hline LysR family transcriptional regulator & 187919777 & Supplemental Figure 6 & 2 aa del & $260-294$ & Clade II \\
\hline
\end{tabular}

a The region of the specified protein that contains the indel. 
A

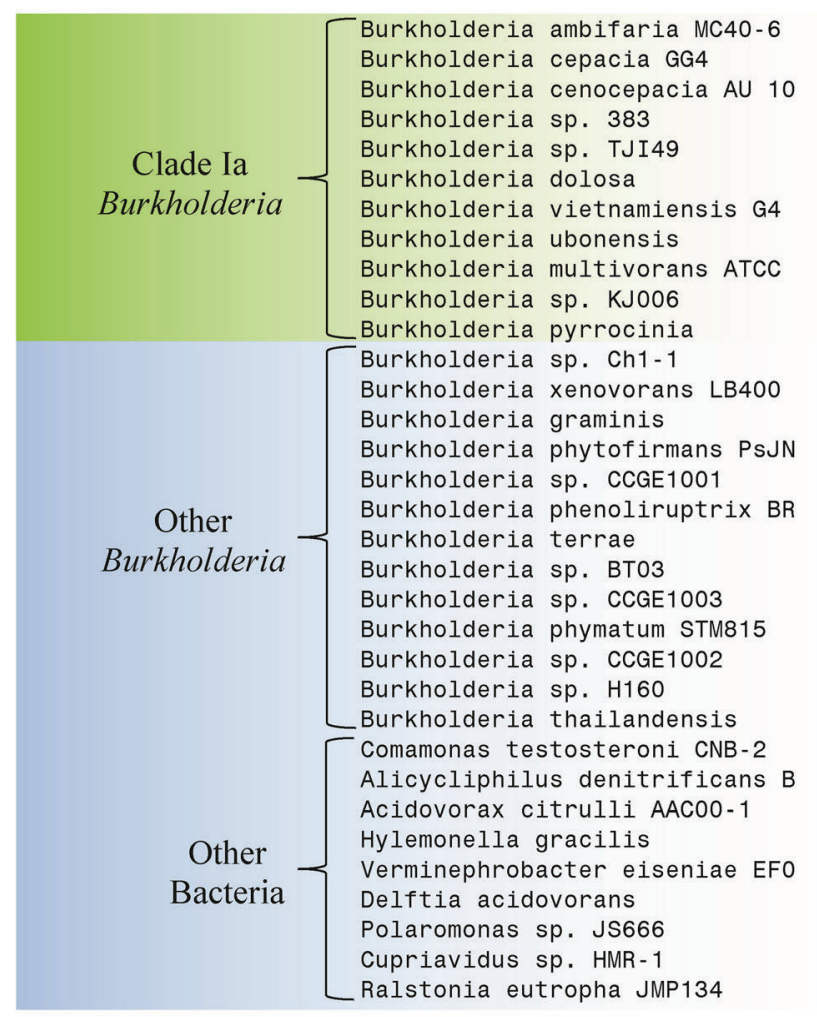

B

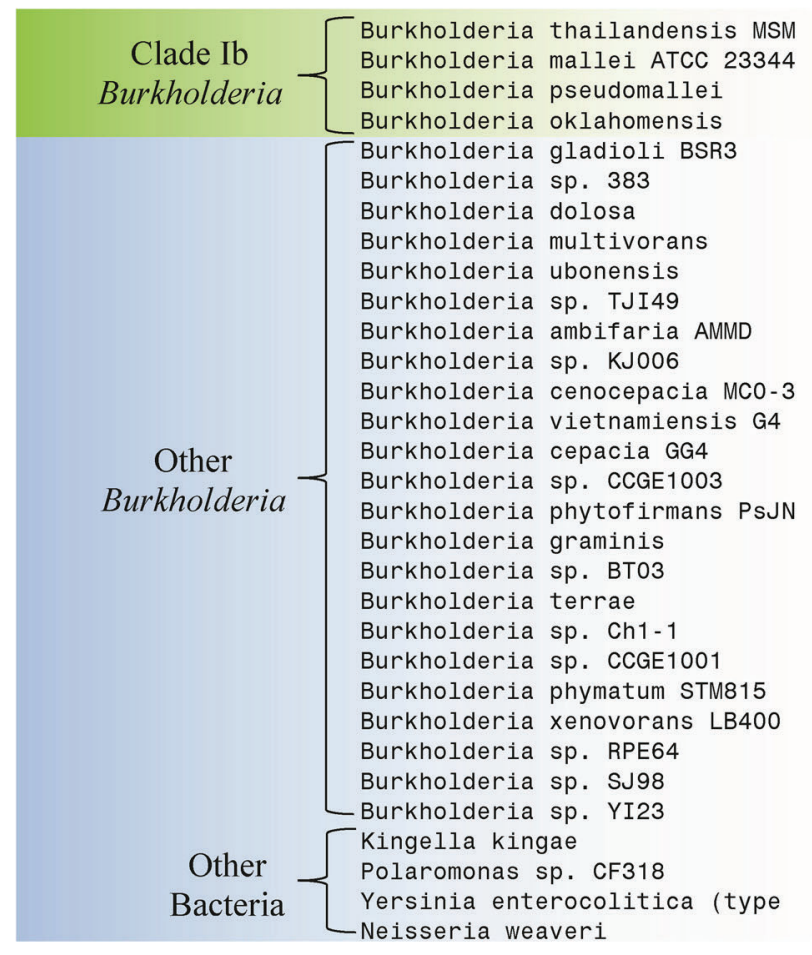

172064454 402570387 107027579 78060928 497380287 493819116 134292445 497780720 161519778 387904119 515900394 494315769 91778287 492938493 187919544 323529857 407710689 494863368 495019334 307727662 186473897 295699309 496198692 492899232 264676299 319763461 120611629 493342257 121610571 512560547 91786943 495920195 73542402

488606492 53716414 497621103 497806594 330821676 78060968 493819092 493455093 497775972 497378269 115359611 387904089 170737090 134292420 402570412 307727211 187919153 492929768 495017718 497457322 494318459 323528511 186473322 91779339 507526505 495628597 377812736 489887671 495145811 510413109 490411191
157

196

QDFQAEPPSEYLFNNVSH YE LEIEHVVDASLPTSEQARLL

-

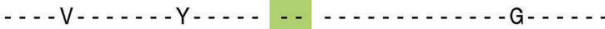

_. -VS - .

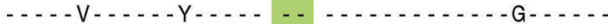

- - EV - .

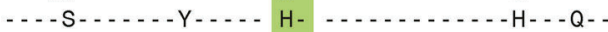

_. RQ

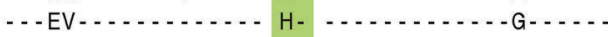

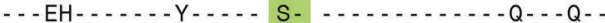

-.-S-IR-...-EI-PA HDV-...-G-.-RAE-E-.

-.-S-IR -...-EI-PA HDV -...-G-.-RAE-E - .

-.-S-IR -..--YET-PA HDV- - I - - G-.-PAE-E - .

-.-S-MR-...-EI-PA HDV-...-G-.-RAE-E..

-.-S-IR -..-YET-PA HDV- - I - - H - - QAE-E - -

-. -A-IR -..-YET-PA HDV- - I-.-H-.-QAE-E -

-.-A-VR -...-SV-PA HDV -...-G--SRAE-E -

-.-A-VR-..--SV-PA HDV-...-G--SRAE-E-

-. -STIR-...-YET-PA HDV-_I-.-G-.-RAE-E - .

-. -STIR-...-.SV-PA HDV-...-G--GRAE-E - .

H--S-LK-..-LTA-PM HDL-....-A-.PAE-E.

H--S-LK-..-LTV-PM HDL -...-A-.-PAE-G- -

-..NTIR-...-YST-PL G-V-....-G-V-ATE-T. .

-.-S-VQ- -V- -VR--QY DQ-...-I-.-A ....

- - AQLQ - - F-VR--PY DQM-...-V - PQ

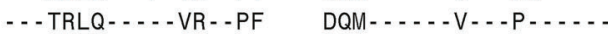

- - RQQQ - . - VR - - PF D D - . - - V - - A - - QQ -

- - ARM - . - - -VR - -PF DQ - . - - VM-GAR - A -

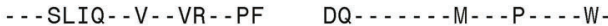

- . - TL-Q- - DF-VRT-LF DQM-...-V-.-R - - A -

-. -SGTK-G - - LR - -PY DQV - ...-ISA-P . -AQ -

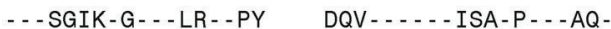

332

EVPMYGLMPKGVKGVQ R PFTPDWASWPMARRVDYAKNLLKO

EVPUYGLPKGVKGQ

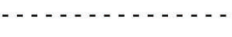

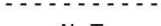

K- $-\mathrm{K}-\mathrm{N}-\mathrm{T}-\mathrm{T}-\mathrm{C}$

$-\mathrm{K}-\ldots-\mathrm{N}-\mathrm{T}-\ldots$

-K- . - . - N-T - . -

$-\mathrm{K}-\ldots-\mathrm{-}-\mathrm{N}-\mathrm{T}-\ldots$

$-\mathrm{K}-\mathrm{F}-\mathrm{-}-\mathrm{N}-\mathrm{T}-\mathrm{-}-\mathrm{C}$

$-K----N-T--A-$

$-\mathrm{K}-\ldots-\mathrm{N}-\mathrm{T}-\mathrm{-}-\mathrm{R}$

$-K-\ldots-N-T-\ldots$

$-\mathrm{K}-\ldots-\mathrm{N}-\mathrm{T}-\mathrm{-R}$

-K- - - - - N-T- - AK

- L - - - - IS - - TQ - AA

- . - - IA- - TE-SG

- L - . - - IS - - TQ - AD

- L - . - - IS - - TE - AA

- L - . - - IS - - TE - AA

- L - - - - IS - - TQ - AA

- L - . - - IS - TQ - AA

- L - . - - IS - TE - AA

- L. - . - IS - - TQ - AA

QI - L-SVL - . - S-GN

QT - L - - - L - . - S S AD

QT - L - - L - . - -S -AD

- TAA - EFT - PAAQ - MK

QT - A - VIV - - TS - AD

QI - A - - FT - TFTE - AN

- TAA - QFT - PAAQ - MK

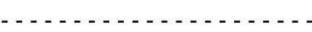

$$
\begin{aligned}
& \text { - - - - - - GK- -E- - -S- . - } \\
& \text {-K- . - A - - - K- . . . . . . } \\
& \text {-. - E - . . - K- - A - . . } \\
& \text { - - -E- _ - _ - - -ET - . . . } \\
& \text { - . - - E - . . . - - K- -ET - . . . . . } \\
& \text { - - - E- - . - K- -ET - . . - } \\
& \text { - - - E- - . - - K- - ET - . . . . } \\
& \text { - - - - K- - AT - D - . } \\
& \text { Y - . - . - - K- - AA - - D - . - } \\
& \text { - . - E - . . - K- IAT - D - . . } \\
& -Y-\ldots \\
& \text { - - - - E- - - - - - K- - ET - - T - . - - } \\
& \text { V-K-1...-K-..-R - - S } \\
& \text { V- - -E- - N- - - K - . - - R - . - } \\
& \text { I-K-E - . . - - K - . - R - . - S } \\
& \text { V-K-E-D- - - K- -E- -R- - - E } \\
& \text { V-K-E- - . - - K- -E- -R - - - E } \\
& \text { V-K- - -ST - - PK- - - - R - . - - } \\
& \text { V-K- - . - - EK- -E- -R- - -S } \\
& \text { V-K-E- . - . - - K- - - T-R - . - E } \\
& \text { V-K - - ST _ - PK - . . RD - . - } \\
& \text { VT-Y- - . - - - K- -EE- -K- -D - } \\
& \text { VSNYE - - T - - - K- -EE- - K- - E - }
\end{aligned}
$$




\section{C

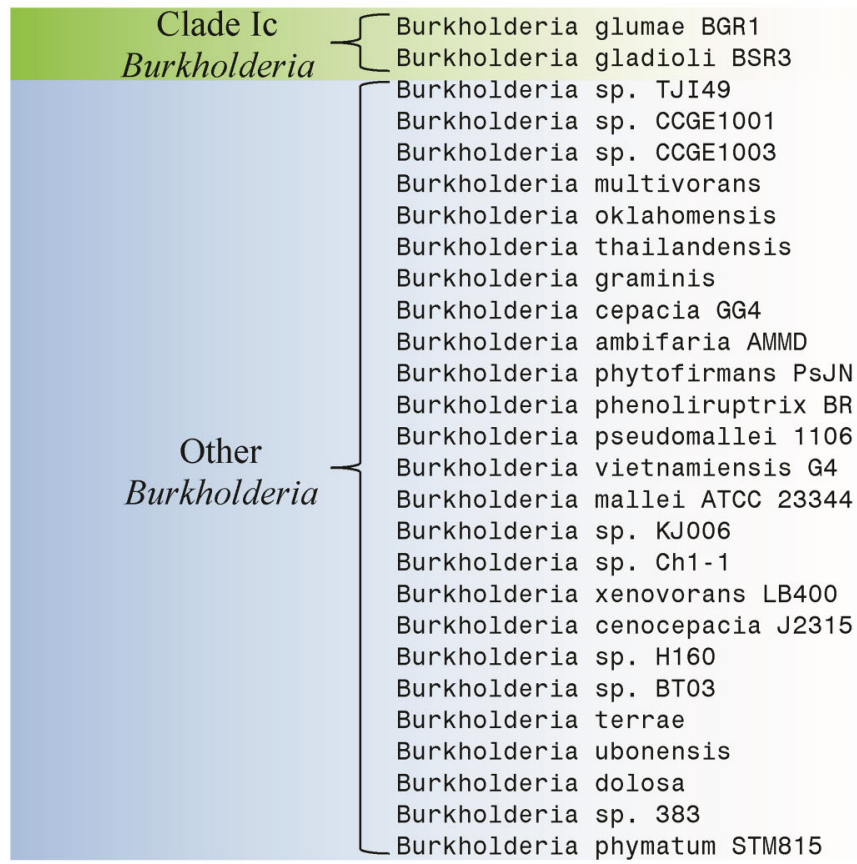

438

238024002

330819826

497381281

323528391

307727331

493458973

497803539

497584362

492930587

402567976

115359353

187919318

407709240

126456001

134292218

53715954

387903880

494318151

91779191

206561957

496197664

495022635

494862840

497783006

493818919

78063931

186471177

403

LVATGQNPNIYNFYHFN P AASGYIAIPDGSLPGKLF

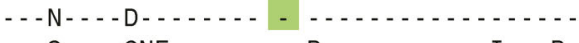

- - -S- - -GNF - . - - P P - - - . - - I - -P -

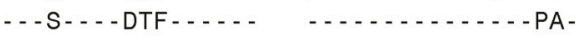

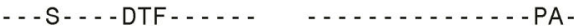

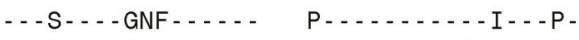

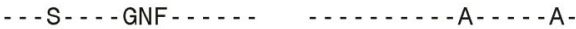

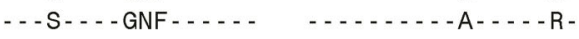

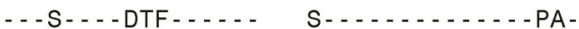

-

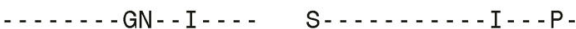

-.-S-..-G-F-AF-. P P-.....-A-I-AA-

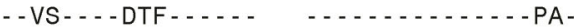

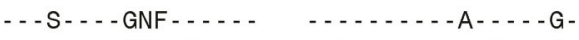

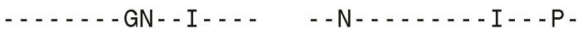

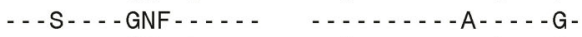

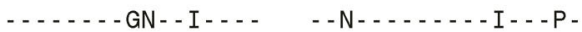

-.-S-..-G-FY-F-.. P P - . - . - A-I - -TA-

-.-S-..-G-FY-F-. $\quad$ P-.....-A-I-AA-

- - A - - - P-FY - . - - -N- - - . - I - - PA-

- - S - . - DTFY-F_. - - N - . - A AT - - TA

- - S- - -DTFY-F- - - -N- - - - AT - - TA -

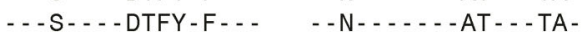

- - A- - - P- -Y-F- - - - N- - - - -A-I - -PA-

- - - - - - GNF - - Y - T P-T - . - -A- - I - - P -

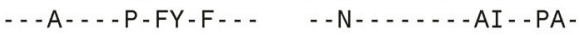

-.-S-.-A-FY-F--E PST-.-V--A-I--SA-
FIGURE 4 | Partial sequence alignments of (A) a histidine utilization repressor showing a 2 amino acid insertion (boxed) identified in all members of the Burkholderia cepacia complex (Clade la) within the genus Burkholderia (B) a periplasmic oligopeptide-binding protein showing a 1 amino acid insertion (boxed) identified in all members of the Burkholderia pseudomallei group (Clade lb) within the genus Burkholderia (C) a SMP-30/gluconolaconase/LRE-like region-containing protein showing a 1 amino acid insertion (boxed) identified in all members of the phytopathogenic Burkholderia clade (Clade Ic). These CSIs were not found in the sequence homologs of these proteins from any other sequenced bacteria in the top 250 BLAST hits. Sequence information for other CSIs specific to subclades within Clade I of the genus Burkholderia are presented in Supplemental Figures $7-15$ and their characteristics are summarized in Table $\mathbf{3}$.
The second group within Clade II of the Burkholderia (Clade IIb), is comprised of a large variety of environmental Burkholderia species (Coenye and Vandamme, 2003; Suarez-Moreno et al., 2012). Our analysis has identified 6 CSIs that are specific to this large group of Burkholderia species. One example of a CSI specific to the members of Clade IIb of the genus Burkholderia is shown in Figure 5B. The CSI consists of a one amino acid insertion in 4-hydroxyacetophenone monooxygenase, which is only present in members of Clade IIb of the genus Burkholderia and not in protein homologs from any other sequenced bacterial group. Information for other 5 CSIs which are specific to members of Clade IIb of the genus Burkholderia are shown in Supplemental Figures 31-35 and their characteristics are summarized in Table 3.

\section{DISCUSSION}

The genus Burkholderia is one of the largest groups of species within the class Betaproteobacteria (Palleroni, 2005; Parte, 2013). The genus contains a variety of bacteria that inhabit a wide range of ecological niches including a number of bacteria that have pathogenic potential (Yabuuchi et al., 1992; Coenye and Vandamme, 2003; Mahenthiralingam et al., 2005; Palleroni, 2005; Compant et al., 2008). The phylogeny of the genus Burkholderia has been studied using a wide array of methodologies based on phenotypic, biochemical, genetic, and genomic characteristics (Stead, 1992; Gillis et al., 1995; Payne et al., 2005; Tayeb et al.,
2008; Onofre-Lemus et al., 2009; Spilker et al., 2009; Ussery et al., 2009; Gyaneshwar et al., 2011; Vandamme and Dawyndt, 2011; Zhu et al., 2011; Estrada-de los Santos et al., 2013). These studies have provided novel insights into the evolutionary relationship of the species within the genus Burkholderia. However, no taxonomic changes have been made to date due to a lack of discrete, distinguishing characteristics identified for the different phylogenetic lineages within the genus (Estrada-de los Santos et al., 2013).

In the present work, we have outlined two major groups of species within the genus Burkholderia: Clade I, which contains all pathogenic members of the genus, and Clade II, which contains a large variety of environmental species. These two groups were found to branch distinctly in a highly resolved phylogenetic tree based on a large number of concatenated protein sequences produced in this work (Figure 1). Evidence for the distinctness of Clade I organisms from other Burkholderia species has been observed in a wide range of previous phylogenetic studies (Payne et al., 2005; Tayeb et al., 2008; Yarza et al., 2008; Spilker et al., 2009; Ussery et al., 2009; Gyaneshwar et al., 2011; Vandamme and Dawyndt, 2011; Zhu et al., 2011; SuarezMoreno et al., 2012; Estrada-de los Santos et al., 2013; Segata et al., 2013). Importantly, we have also identified 6 and 2 CSIs that serve as discrete molecular characteristics of Clade I and Clade II, respectively (Figure 6 and Table 2). These CSIs are the 
Table 3 | Conserved signature indels specific for groups within Clades I and II.

\begin{tabular}{|c|c|c|c|c|c|}
\hline Protein Name & GI Number & Figures & Indel size & Indel position ${ }^{a}$ & Specificity \\
\hline Histidine utilization repressor & 172064454 & Figure 4A & 2 aa ins & $157-196$ & Clade la \\
\hline Molybdate $A B C$ transporter substrate-binding protein & 189352411 & Supplemental Figure 7 & 1 aa ins & $110-158$ & Clade la \\
\hline Acid phosphatase & 221203041 & Supplemental Figure 8 & 1 aa ins & $305-338$ & Clade la \\
\hline Periplasmic oligopeptide-binding protein & 488606492 & Figure 4B & 1 aa ins & $332-372$ & Clade Ib \\
\hline OpgC protein & 53716883 & Supplemental Figure 9 & 1 aa ins & $137-204$ & Clade Ib \\
\hline Polysaccharide deacetylase family protein & 167725414 & Supplemental Figure 10 & 1 aa ins & $29-63$ & Clade Ib \\
\hline Thioredoxin domain protein & 497613277 & Supplemental Figure 11 & 1 aa ins & $247-294$ & Clade Ib \\
\hline SMP-30/gluconolaconase/LRE-like region-containing protein & 238024002 & Figure 4C & 1 aa ins & $403-438$ & Clade Ic \\
\hline Cation efflux protein & 330820376 & Supplemental Figure 12 & 1 aa ins & $129-160$ & Clade Ic \\
\hline putative peptidoglycan-binding LysM/M23B peptidase & 238024763 & Supplemental Figure 13 & 1 aa ins & $155-198$ & Clade Ic \\
\hline SMP-30/gluconolaconase/LRE-like region-containing protein & 238024002 & Supplemental Figure 14 & 2 aa del & $80-130$ & Clade Ic \\
\hline hypothetical protein bgla_2g22890 & 330821370 & Supplemental Figure 15 & 1 aa ins & $322-358$ & Clade Ic \\
\hline 3-phosphoglycerate dehydrogenase & 494056927 & Figure 5A & 1 aa ins & $61-100$ & Clade Ila \\
\hline Hypothetical protein BYI23_A021470 & 377821591 & Supplemental Figure 16 & 1 aa del & $16-76$ & Clade Ila \\
\hline Prepilin peptidase & 377821714 & Supplemental Figure 17 & 1 aa ins & $179-230$ & Clade Ila \\
\hline Uracil-DNA glycosylase & 495619839 & Supplemental Figure 18 & 2 aa ins & $191-230$ & Clade Ila \\
\hline Hypothetical protein BYI23_A015260 & 377820970 & Supplemental Figure 19 & 2 aa ins & $221-270$ & Clade Ila \\
\hline Carboxylate-amine ligase & 377822128 & Supplemental Figure 20 & 1 aa del & $321-362$ & Clade Ila \\
\hline NADH:ubiquinone oxidoreductase subunit M & 494056355 & Supplemental Figure 21 & 3 aa ins & $303-348$ & Clade Ila \\
\hline NADH:ubiquinone oxidoreductase subunit L & 494056354 & Supplemental Figure 22 & 1 aa ins & $538-585$ & Clade Ila \\
\hline ABC transporter & 377821271 & Supplemental Figure 23 & 1 aa del & $59-99$ & Clade Ila \\
\hline Hypothetical protein BYI23_A002220 & 377819666 & Supplemental Figure 24 & 2 aa ins & $133-172$ & Clade Ila \\
\hline 16S rRNA-processing protein RimM & 494056031 & Supplemental Figure 25 & 1 aa ins & $147-201$ & Clade Ila \\
\hline FAD linked oxidase domain-containing protein & 377819737 & Supplemental Figure 26 & 1 aa ins & $106-144$ & Clade Ila \\
\hline Preprotein translocase subunit SecD & 495626933 & Supplemental Figure 27 & 1 aa del & $306-341$ & Clade Ila \\
\hline Mechanosensitive ion channel protein MscS & 494057445 & Supplemental Figure 28 & 3 aa ins & $101-143$ & Clade Ila \\
\hline Hypothetical protein BYI23_A006130 & 377820057 & Supplemental Figure 29 & 1 aa ins & $199-253$ & Clade Ila \\
\hline Uroporphyrinogen-III synthase & 494056428 & Supplemental Figure 30 & 7 aa ins & $37-79$ & Clade Ila \\
\hline 4-hydroxyacetophenone monooxygenase & 496202984 & Figure 5B & 1 aa ins & $380-449$ & Clade IIb \\
\hline Transposase A-like protein & 187923943 & Supplemental Figure 31 & 1 aa ins & $5-50$ & Clade IIb \\
\hline Group 1 glycosyl transferase & 186475830 & Supplemental Figure 32 & 1 aa ins & $153-194$ & Clade IIb \\
\hline 4-hydroxyacetophenone monooxygenase & 496202984 & Supplemental Figure 33 & 3 aa ins & $145-219$ & Clade IIb \\
\hline Undecaprenyl-phosphate glucose phosphotransferase & 209521823 & Supplemental Figure 34 & 1 aa ins & $208-275$ & Clade IIb \\
\hline putative flavin-binding monooxygenase-like protein & 186476032 & Supplemental Figure 35 & 3 aa ins & $102-148$ & Clade IIb \\
\hline
\end{tabular}

a The region of the specified protein that contains the indel.

first discrete features that have been identified that are unique to either Clade I or Clade II of the genus Burkholderia. These CSIs act as independent verification of the phylogenetic trends identified in this and other studies and provide clear evidence that the species from the Clade I are distinct from all other Burkholderia and that they are derived from a common ancestor exclusive of all other Burkholderia. Although sequence information for Clade II members is at present somewhat limited, based upon the shared presence of two CSIs by them, it is likely that they are also derived from a common ancestor exclusive of other bacteria.

Additionally, we have identified molecular evidence, in the form of large numbers of CSIs, which support the distinctiveness of several smaller groups within the genus Burkholderia. The most important of these groups, the $B$. cepacia complex (BCC; Clade Ia) and the B. pseudomallei group (Clade Ib), are supported by the 3 and 4 of the identified CSIs, respectively. The BCC are a group of opportunistic pathogens which colonize immunodificient human hosts and are among the most prevalent and lethal infections in cystic fibrosis patients (Mahenthiralingam et al., 2002, 2005; Biddick et al., 2003; Hauser et al., 2011). The 17 species that make up the BCC are closely related and form a tight monophyletic cluster within the genus Burkholderia (Vandamme and Dawyndt, 2011). The B. pseudomallei group consists of 4 closely related species: $B$. pseudomallei, the causative agent of the highly lethal septicemia melioidosis (White, 2003; Limmathurotsakul and Peacock, 2011), B. mallei, the causative agent of the equine disease glanders and occasional human infections (Whitlock et al., 2007), and the largely non-pathogenic organisms, Burkholderia thailandensis and Burkholderia oklahomensis (Deshazer, 2007). The identified CSIs are highly specific characteristics of these two important pathogenic groups and they 
A

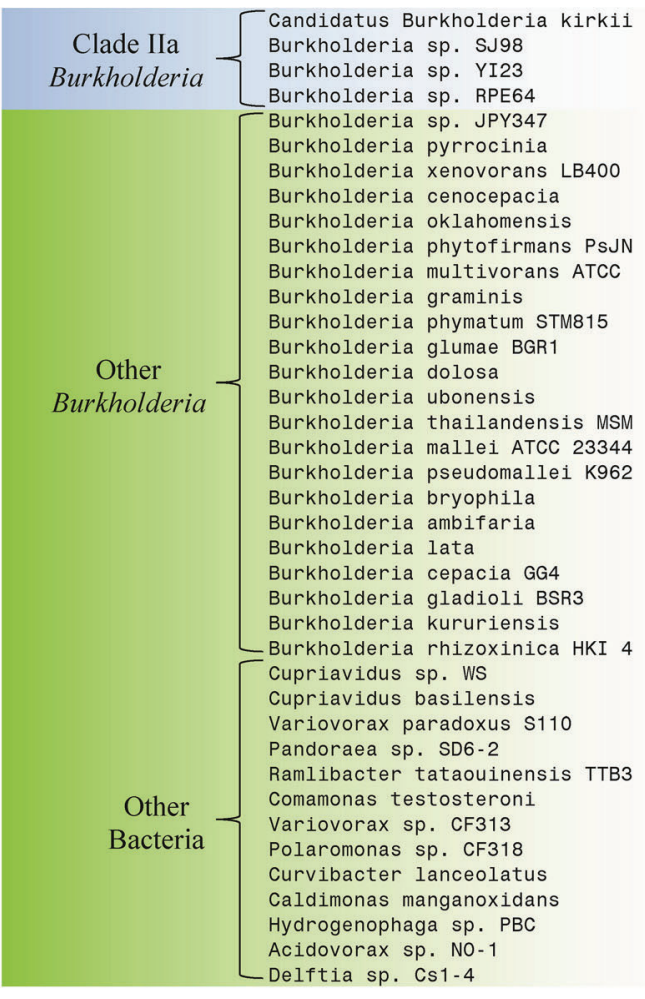

B

494056927
495627620
377819458
507514550
517252484
515904055
91781423
493541519
497786533
187922310
161526298
492932713
186474800
238028950
493815470
497776121
488604417
53724668
53717757
518911408
493802193
78067952
402565104
330818693
516382324
312797609
519050400
493151034
239815615
498504991
337279845
489166115
495112131
495147651
518403185
518390521
497204968
496179791
333914892

61

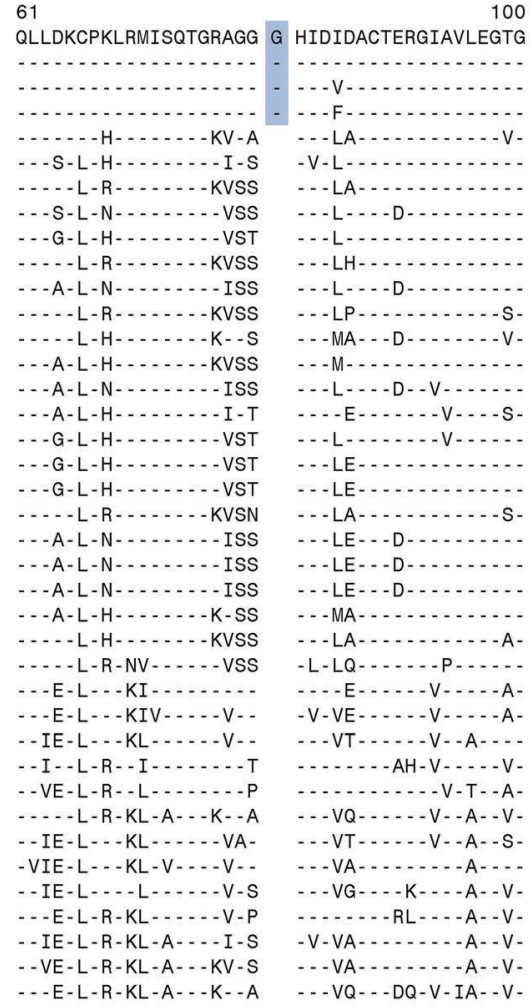

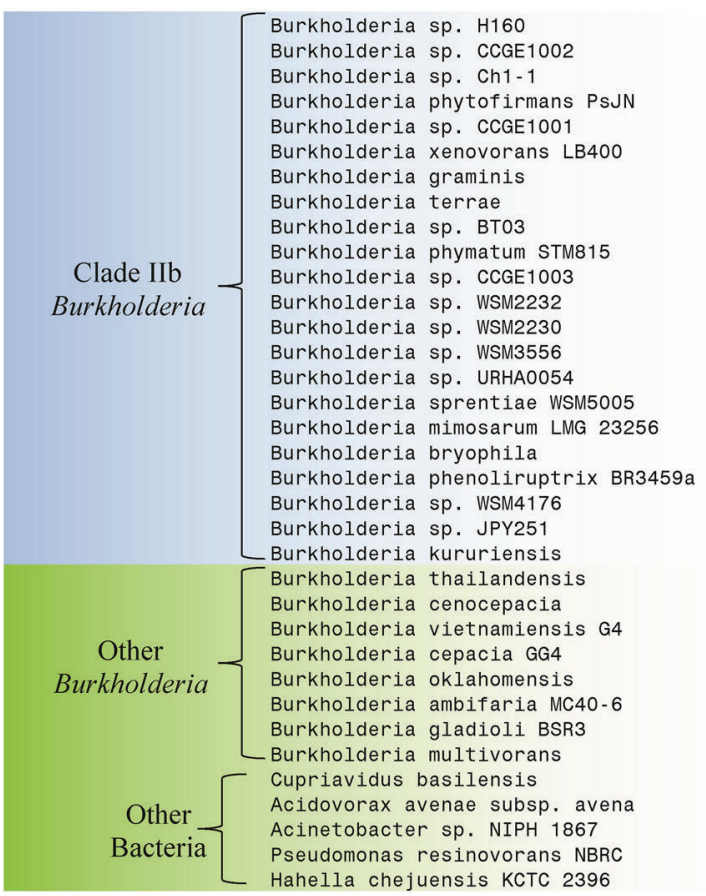

496202984 295676766 494327703 187924282 323525825 91783855 492936566 494862209 495008604 186476032 307729928 548691109 548606735 548691174 522812216 548699263 548694927 518914327 407713185 517228895 517246749 516386272 497584847 493525653 134291654 402569879 497808405 172062537 330821758 493454498 493139021 326316554 491354526 512617894 83647045
380 GTGFLVNDVRAPFEVTGLGGTDLSTLW - - - P - . -..Q-D....-D-A-..Q- Q- Q - - G-D-D-A- - GA- - - Q - . - G- - D - - -V- - A- - GA- - - Q Q - - G - D - . - -A-GA -..-Q-..-G- -D - . . -A- -GA-. - Q-...-G- - D-A-I - -A- -GA- -..-Q-...GP - -D - - I - -A - -G - . - . - . - - GPA- - - - I - -A- - G- . - - - QF - . -GP - - D - - - V - -A - -GA -. - QR-G-G- . . . . IS-AN-RA- . - - - Q - . -G- -D - - V- -A- -GA - . Q Q . . P . . . . - D - E . . . -..-Q-..-D- - - Q - . I - - Q- - G-M- - - QF - GFG - . - . - - I - -A - -RA- - - Q- - - - - - - -

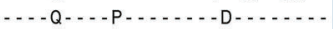
- . - Q - . P . . . . D D . . . -..-H-..-G-...-.V- -A- - GA- . - AATEFL - MHIV-RA-VT - NDA- - - AATEFLS - MRI - -R - - L - - NDA - . - - AATEFLS - MRI - -RD - L - - NDA - . - - AATSFLS - MRI - - RN - L - -NDA- - - AATEFL - MRIV - RA-VT - NDA- - - AATAFLS - MRI - - RD - R - -NDA- - - -AATEFLS-MRI - -R - - LE - NEV -..-Q-A-PFPRGAIV-R - - L-IVDA- - - QAT - FL - MR - . - - D - I - - NQA - - - AATEFLS - MKI - -R - - LE - NAA- - - AATEFM - - MQI - - - N-KR - KDQ- . - - AAT - FL - - MSIR - . - - RE -NDAA - . QAAEAVI - - DL I . . . - VS - NQA . . . .

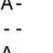




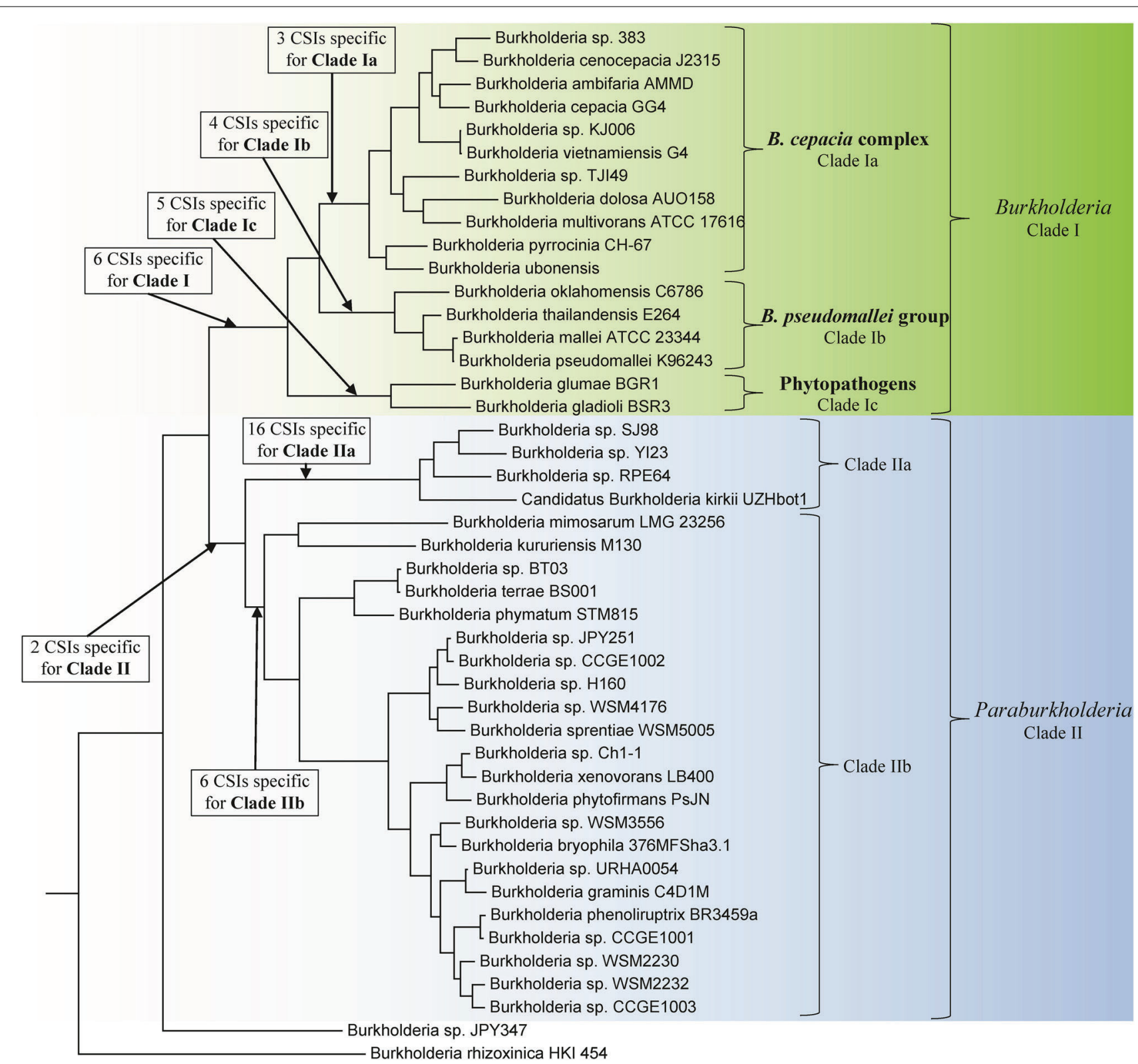

FIGURE 6 | A summary diagram depicting the distribution of identified CSIs and the proposed names of the two major groups (Clade I and II) within Burkholderia. The major Burkholderia clades are indicated by brackets and highlighting.

provide novel and useful targets for the development of diagnostic assays for either the BCC or the B. pseudomallei group (Ahmod et al., 2011; Wong et al., 2014). We have identified CSIs for three other groups within the genus Burkholderia: A group of plant pathogenic Burkholderia related to the BCC and B. pseudomallei group (Clade Ic), a group containing unnamed and candidate Burkholderia species (Clade IIa), and a group consisting of environmental Burkholderia (Clade IIb). We have identified 6, 16, and 6 CSIs for these three groups, respectively. These CSIs provide important differentiating characteristics for these groups, particularly for Clades IIa and IIb which are related groups that have no other identified differentiating characteristics (Suarez-Moreno et al., 2012).
The phylogenetic analyses, identified CSIs, and the pathogenic characteristics of the different Burkholderia species presented in this work strongly suggest that the genus Burkholderia is made up of at least two distinct lineages. One lineage consisting of the BCC and related organisms (Clade I) and another consisting of a wide range of environmental organisms (Clade II). This latter clade is phylogenetically highly diverse and there is a paucity of sequence information available for its members. Thus, it is possible that in future this latter clade may be found to consist of more than one distinct bacterial lineage, however, it is currently clear that Clade I and Clade II represent distinct lineages. Evidence for the distinctness of the Clade I members from other Burkholderia species has been identified in 
Table 4 | Descriptions of the new combinations in the genus Paraburkholderia gen. nov.

\begin{tabular}{|c|c|c|c|}
\hline New Combination & Basonym & Type Strain & References \\
\hline \multirow[t]{3}{*}{ Paraburkholderia acidipaludis comb. nov. } & Burkholderia acidipaludis & SA33 & Aizawa et al., 2010b \\
\hline & & NBRC 101816 & \\
\hline & & VTCC-D6-6 & \\
\hline \multirow{2}{*}{$\begin{array}{l}\text { Candidatus Paraburkholderia } \\
\text { andongensis comb. nov. }\end{array}$} & Candidatus Burkholderia & - & Lemaire et al., 2011 \\
\hline & andongensis & & \\
\hline \multirow{10}{*}{$\begin{array}{l}\text { Paraburkholderia andropogonis comb. } \\
\text { nov. }\end{array}$} & Burkholderia andropogonis & ATCC 23061 & Gillis et al., 1995 \\
\hline & & CCUG 32772 & \\
\hline & & CFBP 2421 & \\
\hline & & CIP 105771 & \\
\hline & & DSM 9511 & \\
\hline & & ICMP 2807 & \\
\hline & & JCM 10487 & \\
\hline & & LMG 2129 & \\
\hline & & NCPPB 934 & \\
\hline & & NRRL B-14296 & \\
\hline \multirow[t]{3}{*}{ Paraburkholderia aspalathi comb. nov. } & Burkholderia aspalathi & VG1C & Mavengere et al., 2014 \\
\hline & & DSM 27239 & \\
\hline & & LMG 27731 & \\
\hline \multirow[t]{3}{*}{ Paraburkholderia bannensis comb. nov. } & Burkholderia bannensis & E25 & Aizawa et al., 2011 \\
\hline & & BCC 36998 & \\
\hline & & NBRC 103871 & \\
\hline \multirow[t]{3}{*}{ Paraburkholderia bryophila comb. nov. } & Burkholderia bryophila & $1 \mathrm{~S} 18$ & Vandamme et al., 2007 \\
\hline & & CCUG 52993 & \\
\hline & & LMG 23644 & \\
\hline \multirow[t]{3}{*}{ Paraburkholderia caballeronis comb. nov. } & Burkholderia caballeronis & TNe-841 & Martínez-Aguilar et al., 2013 \\
\hline & & CIP 110324 & \\
\hline & & LMG 26416 & \\
\hline \multirow[t]{6}{*}{ Paraburkholderia caledonica comb. nov. } & Burkholderia caledonica & W50D & Coenye et al., 2001a \\
\hline & & CCUG 42236 & \\
\hline & & CIP 107098 & \\
\hline & & JCM 21561 & \\
\hline & & LMG 19076 & \\
\hline & & NBRC 102488 & \\
\hline Candidatus Paraburkholderia calva comb. & Candidatus Burkholderia calva & - & Van Oevelen et al., 2004 \\
\hline nov. & & & \\
\hline \multirow[t]{5}{*}{ Paraburkholderia caribensis comb. nov. } & Burkholderia caribensis & MWAP64 & Achouak et al., 1999 \\
\hline & & CCUG 42847 & \\
\hline & & CIP 106784 & \\
\hline & & DSM 13236 & \\
\hline & & LMG 18531 & \\
\hline \multirow[t]{8}{*}{ Paraburkholderia caryophylli comb. nov. } & Burkholderia caryophylli & ATCC 25418 & Yabuuchi et al., 1992 \\
\hline & & CCUG 20834 & \\
\hline & & CFBP 2429 & \\
\hline & & CFBP 3818 & \\
\hline & & CIP 105770 & \\
\hline & & DSM 50341 & \\
\hline & & HAMBI 2159 & \\
\hline & & ICMP 512 & \\
\hline
\end{tabular}


Table 4 | Continued

\begin{tabular}{|c|c|c|c|}
\hline New Combination & Basonym & Type Strain & References \\
\hline & & JCM 9310 & \\
\hline & & JCM 10488 & \\
\hline & & LMG 2155 & \\
\hline & & NCPPB 2151 & \\
\hline \multirow[t]{2}{*}{ Paraburkholderia choica comb. nov. } & Burkholderia choica & LMG 22940 & Vandamme et al., 2013 \\
\hline & & CCUG 63063 & \\
\hline \multirow[t]{3}{*}{ Paraburkholderia denitrificans comb. nov. } & Burkholderia denitrificans & KIS30-44 & Lee et al., 2012 \\
\hline & & DSM 24336 & \\
\hline & & KACC 12733 & \\
\hline \multirow{5}{*}{$\begin{array}{l}\text { Paraburkholderia diazotrophica comb. } \\
\text { nov. }\end{array}$} & Burkholderia diazotrophica & JPY461 & Sheu et al., 2013 \\
\hline & & NKMU-JPY461 & \\
\hline & & BCRC 80259 & \\
\hline & & KCTC 23308 & \\
\hline & & LMG 26031 & \\
\hline \multirow[t]{3}{*}{ Paraburkholderia dilworthii comb. nov. } & Burkholderia dilworthii & WSM3556 & De Meyer et al., 2014 \\
\hline & & LMG 27173 & \\
\hline & & HAMBI 3353 & \\
\hline \multirow[t]{3}{*}{ Paraburkholderia eburne comb. nov. } & Burkholderia eburne & RR11 & Kang et al., 2014 \\
\hline & & KEMC 7302-065 & \\
\hline & & JCM 18070 & \\
\hline \multirow{3}{*}{$\begin{array}{l}\text { Paraburkholderia endofungorum comb. } \\
\text { nov. }\end{array}$} & Burkholderia endofungorum & HKI 456 & Partida-Martinez et al., 2007 \\
\hline & & CIP 109454 & \\
\hline & & DSM 19003 & \\
\hline \multirow[t]{4}{*}{ Paraburkholderia ferrariae comb. nov. } & Burkholderia ferrariae & FeGI01 & Valverde et al., 2006 \\
\hline & & CECT 7171 & \\
\hline & & DSM 18251 & \\
\hline & & LMG 23612 & \\
\hline \multirow[t]{6}{*}{ Paraburkholderia fungorum comb. nov. } & Burkholderia fungorum & Croize P763-2 & Coenye et al., 2001a \\
\hline & & CCUG 31961 & \\
\hline & & CIP 107096 & \\
\hline & & JCM 21562 & \\
\hline & & LMG 16225 & \\
\hline & & NBRC 102489 & \\
\hline \multirow[t]{3}{*}{ Paraburkholderia ginsengisoli comb. nov. } & Burkholderia ginsengisoli & KMY03 & Kim et al., 2006 \\
\hline & & КСТC 12389 & \\
\hline & & NBRC 100965 & \\
\hline \multirow[t]{6}{*}{ Paraburkholderia glathei comb. nov. } & Burkholderia glathei & ATCC 29195 & Vandamme et al., 1997 \\
\hline & & CFBP 4791 & \\
\hline & & CIP 105421 & \\
\hline & & DSM 50014 & \\
\hline & & JCM 10563 & \\
\hline & & LMG 14190 & \\
\hline \multirow[t]{5}{*}{ Paraburkholderia graminis comb. nov. } & Burkholderia graminis & C4D1M & Viallard et al., 1998 \\
\hline & & ATCC 700544 & \\
\hline & & CCUG 42231 & \\
\hline & & CIP 106649 & \\
\hline & & LMG 18924 & \\
\hline
\end{tabular}


Table 4 | Continued

\begin{tabular}{|c|c|c|c|}
\hline New Combination & Basonym & Type Strain & References \\
\hline Paraburkholderia grimmiae comb. nov. & Burkholderia grimmiae & $\begin{array}{l}\text { R27 } \\
\text { CGMCC } 1.11013 \\
\text { DSM } 25160\end{array}$ & Tian et al., 2013 \\
\hline Paraburkholderia heleia comb. nov. & Burkholderia heleia & $\begin{array}{l}\text { SA41 } \\
\text { NBRC } 101817 \\
\text { VTCC-D6-7 }\end{array}$ & Aizawa et al., 2010a \\
\hline $\begin{array}{l}\text { Candidatus Paraburkholderia hispidae } \\
\text { comb. nov. }\end{array}$ & $\begin{array}{l}\text { Candidatus Burkholderia } \\
\text { hispidae }\end{array}$ & - & Lemaire et al., 2012 \\
\hline Paraburkholderia hospita comb. nov. & Burkholderia hospita & $\begin{array}{l}\text { LMG } 20598 \\
\text { CCUG } 43658\end{array}$ & Goris et al., 2002 \\
\hline Paraburkholderia humi comb. nov. & Burkholderia humi & $\begin{array}{l}\text { LMG } 22934 \\
\text { CCUG } 63059\end{array}$ & Vandamme et al., 2013 \\
\hline $\begin{array}{l}\text { Candidatus Paraburkholderia kirkii comb. } \\
\text { nov. }\end{array}$ & Candidatus Burkholderia kirkii & - & Van Oevelen et al., 2002a \\
\hline Paraburkholderia kururiensis comb. nov. & Burkholderia kururiensis & $\begin{array}{l}\text { KP23 } \\
\text { ATCC } 700977 \\
\text { CCUG } 43663 \\
\text { CIP } 106643 \\
\text { DSM } 13646 \\
\text { JCM } 10599 \\
\text { LMG } 19447\end{array}$ & Zhang et al., 2000 \\
\hline $\begin{array}{l}\text { Paraburkholderia megapolitana comb. } \\
\text { nov. }\end{array}$ & Burkholderia megapolitana & $\begin{array}{l}\text { A3 } \\
\text { CCUG } 53006 \\
\text { LMG } 23650\end{array}$ & Vandamme et al., 2007 \\
\hline Paraburkholderia mimosarum comb. nov. & Burkholderia mimosarum & $\begin{array}{l}\text { PAS44 } \\
\text { BCRC } 17516 \\
\text { LMG } 23256\end{array}$ & Chen et al., 2006 \\
\hline $\begin{array}{l}\text { Candidatus Paraburkholderia } \\
\text { nigropunctata comb. nov. }\end{array}$ & $\begin{array}{l}\text { Candidatus Burkholderia } \\
\text { nigropunctata }\end{array}$ & - & Van Oevelen et al., 2004 \\
\hline Paraburkholderia nodosa comb. nov. & Burkholderia nodosa & $\begin{array}{l}\text { Br3437 } \\
\text { BCRC } 17575 \\
\text { LMG } 23741\end{array}$ & Chen et al., 2007 \\
\hline Paraburkholderia oxyphila comb. nov. & Burkholderia oxyphila & $\begin{array}{l}\text { OX-01 } \\
\text { DSM } 22550 \\
\text { NBRC } 105797\end{array}$ & Otsuka et al., 2011 \\
\hline $\begin{array}{l}\text { Candidatus Paraurkholderia petitii comb. } \\
\text { nov. }\end{array}$ & $\begin{array}{l}\text { Candidatus Burkholderia } \\
\text { petitii }\end{array}$ & - & Lemaire et al., 2011 \\
\hline $\begin{array}{l}\text { Paraburkholderia phenazinium comb. } \\
\text { nov. }\end{array}$ & Burkholderia phenazinium & $\begin{array}{l}\text { ATCC } 33666 \\
\text { CCUG } 20836 \\
\text { CFBP } 4793 \\
\text { CIP } 106502 \\
\text { DSM } 10684 \\
\text { JCM } 10564 \\
\text { LMG } 2247 \\
\text { NCIMB } 11027\end{array}$ & Viallard et al., 1998 \\
\hline
\end{tabular}


Table 4 | Continued

\begin{tabular}{|c|c|c|c|}
\hline New Combination & Basonym & Type Strain & References \\
\hline $\begin{array}{l}\text { Paraburkholderia phenoliruptrix comb. } \\
\text { nov. }\end{array}$ & Burkholderia phenoliruptrix & $\begin{array}{l}\text { AC1100 } \\
\text { CCUG } 48558 \\
\text { LMG } 22037\end{array}$ & Coenye et al., 2004 \\
\hline Paraburkholderia phymatum comb. nov. & Burkholderia phymatum & $\begin{array}{l}\text { STM815 } \\
\text { LMG } 21445 \\
\text { CCUG } 47179\end{array}$ & Vandamme et al., 2002a \\
\hline $\begin{array}{l}\text { Paraburkholderia phytofirmans comb. } \\
\text { nov. }\end{array}$ & Burkholderia phytofirmans & $\begin{array}{l}\text { PsJN } \\
\text { CCUG } 49060 \\
\text { LMG } 22146\end{array}$ & Sessitsch et al., 2005 \\
\hline Paraburkholderia rhizoxinica comb. nov. & Burkholderia rhizoxinica & $\begin{array}{l}\text { HKI } 454 \\
\text { CIP } 109453 \\
\text { DSM } 19002\end{array}$ & Partida-Martinez et al., 2007 \\
\hline Paraburkholderia rhynchosiae comb. nov. & Burkholderia rhynchosiae & $\begin{array}{l}\text { WSM3937 } \\
\text { LMG } 27174 \\
\text { HAMBI } 3354\end{array}$ & De Meyer et al., 2013b \\
\hline $\begin{array}{l}\text { Candidatus Paraburkholderia rigidae } \\
\text { comb. nov. }\end{array}$ & $\begin{array}{l}\text { Candidatus Burkholderia } \\
\text { rigidae }\end{array}$ & - & Lemaire et al., 2012 \\
\hline Paraburkholderia sabiae comb. nov. & Burkholderia sabiae & $\begin{array}{l}\text { Br3407 } \\
\text { BCRC } 17587 \\
\text { LMG } 24235\end{array}$ & Chen et al., 2008 \\
\hline Paraburkholderia sacchari comb. nov. & Burkholderia sacchari & $\begin{array}{l}\text { CCT } 6771 \\
\text { CCUG } 46043 \\
\text { CIP } 107211 \\
\text { IPT } 101 \\
\text { LMG } 19450\end{array}$ & Brämer et al., 2001 \\
\hline Paraburkholderia sartisoli comb. nov. & Burkholderia sartisoli & $\begin{array}{l}\text { RP007 } \\
\text { CCUG } 53604 \\
\text { ICMP } 13529 \\
\text { LMG } 24000\end{array}$ & Vanlaere et al., 2008 \\
\hline $\begin{array}{l}\text { Candidatus Paraburkholderia } \\
\text { schumannianae comb. nov. }\end{array}$ & $\begin{array}{l}\text { Candidatus Burkholderia } \\
\text { schumannianae }\end{array}$ & - & Lemaire et al., 2012 \\
\hline $\begin{array}{l}\text { Paraburkholderia sediminicola comb. } \\
\text { nov. }\end{array}$ & Burkholderia sediminicola & $\begin{array}{l}\text { HU2-65W } \\
\text { KCTC } 22086 \\
\text { LMG } 24238\end{array}$ & Lim et al., 2008 \\
\hline Paraburkholderia silvatlantica comb. nov. & Burkholderia silvatlantica & $\begin{array}{l}\text { SRMrh-20 } \\
\text { ATCC BAA-1244 } \\
\text { LMG } 23149\end{array}$ & Perin et al., 2006 \\
\hline Paraburkholderia soli comb. nov. & Burkholderia soli & $\begin{array}{l}\text { GP25-8 } \\
\text { DSM } 18235 \\
\text { KACC } 11589\end{array}$ & Yoo et al., 2007 \\
\hline Paraburkholderia sordidicola comb. nov. & Burkholderia sordidicola & $\begin{array}{l}\text { CCUG } 49583 \\
\text { JCM } 11778 \\
\text { KCTC } 12081\end{array}$ & Lim et al., 2003 \\
\hline Paraburkholderia sprentiae comb. nov. & Burkholderia sprentiae & $\begin{array}{l}\text { WSM5005 } \\
\text { LMG } 27175 \\
\text { HAMBI } 3357\end{array}$ & De Meyer et al., 2013a \\
\hline
\end{tabular}




\section{Table 4 | Continued}

\begin{tabular}{|c|c|c|c|}
\hline New Combination & Basonym & Type Strain & References \\
\hline Paraburkholderia terrae comb. nov. & Burkholderia terrae & $\begin{array}{l}\text { KMY02 } \\
\text { KCTC } 12388 \\
\text { NBRC } 100964\end{array}$ & Yang et al., 2006 \\
\hline Paraburkholderia terrestris comb. nov. & Burkholderia terrestris & $\begin{array}{l}\text { LMG } 22937 \\
\text { CCUG } 63062\end{array}$ & Vandamme et al., 2013 \\
\hline Paraburkholderia tropica comb. nov. & Burkholderia tropica & $\begin{array}{l}\text { Ppe8 } \\
\text { ATCC BAA-831 } \\
\text { DSM } 15359 \\
\text { LMG } 22274\end{array}$ & Reis et al., 2004 \\
\hline Paraburkholderia tuberum comb. nov. & Burkholderia tuberum & $\begin{array}{l}\text { STM678 } \\
\text { CCUG } 47178 \\
\text { LMG } 21444\end{array}$ & Vandamme et al., 2002a \\
\hline Paraburkholderia udeis comb. nov. & Burkholderia udeis & $\begin{array}{l}\text { LMG } 27134 \\
\text { CCUG } 63061\end{array}$ & Vandamme et al., 2013 \\
\hline $\begin{array}{l}\text { Paraburkholderia zhejiangensis comb. } \\
\text { nov. }\end{array}$ & Burkholderia zhejiangensis & $\begin{array}{l}\text { OP-1 } \\
\text { KCTC } 23300\end{array}$ & Lu et al., 2012 \\
\hline
\end{tabular}

a number of previous phylogenetic studies (Payne et al., 2005; Tayeb et al., 2008; Yarza et al., 2008; Spilker et al., 2009; Ussery et al., 2009; Gyaneshwar et al., 2011; Vandamme and Dawyndt, 2011; Zhu et al., 2011; Suarez-Moreno et al., 2012; Estradade los Santos et al., 2013; Segata et al., 2013). Estrada-de los Santos et al. (2013) recently completed a phylogenetic analysis of the genus Burkholderia utilizing the multilocus sequence analysis of $a t p D, g l t B$, lepA, and recA genes in combination with the 16S rRNA gene, which provides compelling evidence for the presence of two distinct evolutionary lineages within the genus Burkholderia. However, these authors have refrained from formally proposing a division of the genus into two genera due to a paucity of differentiating characteristics for the two groups. Our comparative analysis of Burkholderia genomes has identified a set of distinctive molecular characteristics that clearly differentiate the two evolutionary lineages within the genus Burkholderia in addition the phylogenetic evidence. In light of the abundance of phylogenetic and molecular evidence for the presence of two distinct evolutionary lineages within the genus Burkholderia, and the distinct pathogenicity profiles of the members of these two groups, we are proposing that genus Burkholderia should be divided into two separate genera. The first of these monophyletic genera, which comprises of all the clinically relevant species and clearly distinguished from all other Burkholderia species, will retain the name Burkholderia (Clade I). For the remainder of the Burkholderia species (Clade II), which include a wide range of environmental species, we propose the name Paraburkholderia gen. nov. An emended description of the genus Burkholderia and a description of Paraburkholderia gen. nov. are provided below. Brief descriptions of the new species 
combinations within Paraburkholderia gen. nov. are presented in Table 4

\section{EMENDED DESCRIPTION OF THE GENUS BURKHOLDERIA (Yabuuchi et al., 1993 EMEND. Gillis et al., 1995)}

The genus contains the type species B. cepacia (Yabuuchi et al., 1993). The species from this genus are gram-negative, straight or slightly curved rods, which exhibit motility mediated by one or more polar flagella. Only, B. mallei lacks flagella and is nonmotile. The species do not produce sheaths or prosthecae and do not go through any resting stages. Most species are able to accumulate and utilize poly- $\beta$-hydroxybutyrate (PHB) for growth. The species are mostly aerobic chemoorganotrophs, but some species are capable of anaerobic respiration using nitrate as the terminal electron acceptor. The $\mathrm{G}+\mathrm{C}$ content for the members of the genus ranges from 65.7 to $68.5 \%$. The members of the genus form a distinct monophyletic clade in phylogenetic trees, and they are distinguished from all other bacteria by the conserved sequence indels reported in this work in the following proteins: Periplasmic amino acid-binding protein, 4-hydroxybenzoate 3monooxygenase, 6-phosphogluconate dehydrogenase, Sarcosine oxidase subunit alpha, a putative lipoprotein, and a putative lyase (Table 2).

\section{DESCRIPTION OF THE GENUS PARABURKHOLDERIA GEN. NOV.}

The genus contains the type species Paraburkholderia graminis comb. nov. (Basonym: Burkholderia graminis, Viallard et al., 1998) The species from this genus are gram-negative straight or slightly curved rods with one or more polar flagella. Other morphological and metabolic characteristics are similar to genus Burkholderia. The $\mathrm{G}+\mathrm{C}$ content for the members of the genus ranges from 61.4 to $65.0 \%$. The species are not associated with humans. The members of this genus generally form a distinct clade in the neighborhood of genus Burkholderia in phylogenetic trees, and they lack the molecular signatures which are specific for Burkholderia. Most of the sequenced members from this genus contain the conserved sequence indels reported in this work in the protein sequences of an unnamed dehydrogenase and a LysR family transcriptional regulator (Table 2 ).

\section{SUPPLEMENTARY MATERIAL}

The Supplementary Material for this article can be found online at: http://www.frontiersin.org/journal/10.3389/fgene. 2014.00429/abstract

\section{REFERENCES}

Achouak, W., Christen, R., Barakat, M., Martel, M.-H., and Heulin, T. (1999). Burkholderia caribensis sp. nov., an exopolysaccharide-producing bacterium isolated from vertisol microaggregates in Martinique. Int. J. Syst. Bacteriol. 49, 787-794. doi: 10.1099/00207713-49-2-787

Ahmod, N. Z., Gupta, R. S., and Shah, H. N. (2011). Identification of a Bacillus anthracis specific indel in the $y e a C$ gene and development of a rapid pyrosequencing assay for distinguishing B. anthracis from the B. cereus group. J. Microbiol. Methods 87, 278-285. doi: 10.1016/j.mimet.2011.08.015

Aizawa, T., Ve, N. B., Nakajima, M., and Sunairi, M. (2010a). Burkholderia heleia sp. nov., a nitrogen-fixing bacterium isolated from an aquatic plant, Eleocharis dulcis, that grows in highly acidic swamps in actual acid sulfate soil areas of Vietnam. Int. J. Syst. Evol. Microbiol. 60, 1152-1157. doi: 10.1099/ijs.0. 015198-0
Aizawa, T., Ve, N. B., Vijarnsorn, P., Nakajima, M., and Sunairi, M. (2010b). Burkholderia acidipaludis sp. nov., aluminium-tolerant bacteria isolated from Chinese water chestnut (Eleocharis dulcis) growing in highly acidic swamps in South-East Asia. Int. J. Syst. Evol. Microbiol. 60, 2036-2041. doi: 10.1099/ijs.0.018283-0

Aizawa, T., Vijarnsorn, P., Nakajima, M., and Sunairi, M. (2011). Burkholderia bannensis sp. nov., an acid-neutralizing bacterium isolated from torpedo grass (Panicum repens) growing in highly acidic swamps. Int. J. Syst. Evol. Microbiol. 61, 1645-1650. doi: 10.1099/ijs.0.026278-0

Biddick, R., Spilker, T., Martin, A., and LiPuma, J. J. (2003). Evidence of transmission of Burkholderia cepacia, Burkholderia multivorans and Burkholderia dolosa among persons with cystic fibrosis. FEMS Microbiol. Lett. 228, 57-62. doi: 10.1016/S0378-1097(03)00724-9

Brämer, C. O., Vandamme, P., da Silva, L. F., Gomez, J., and Steinbüchel, A. (2001). Polyhydroxyalkanoate-accumulating bacterium isolated from soil of a sugar-cane plantation in Brazil. Int. J. Syst. Evol. Microbiol. 51, 1709-1713. doi: 10.1099/00207713-51-5-1709

Caballero-Mellado, J., Martínez-Aguilar, L., Paredes-Valdez, G., and Estrada-de los Santos, P. (2004). Burkholderia unamae sp. nov., an N2-fixing rhizospheric and endophytic species. Int. J. Syst. Evol. Microbiol. 54, 1165-1172. doi: 10.1099/ijs.0.02951-0

Castresana, J. (2000). Selection of conserved blocks from multiple alignments for their use in phylogenetic analysis. Mol. Biol. Evol. 17, 540-552. doi: 10.1093/oxfordjournals.molbev.a026334

Chain, P. S., Denef, V. J., Konstantinidis, K. T., Vergez, L. M., Agullo, L., Reyes, V. L., et al. (2006). Burkholderia xenovorans LB400 harbors a multi-replicon, 9.73-Mbp genome shaped for versatility. Proc. Natl. Acad. Sci. U.S.A. 103, 15280-15287. doi: 10.1073/pnas.0606924103

Charlebois, R. L., and Doolittle, W. F. (2004). Computing prokaryotic gene ubiquity: rescuing the core from extinction. Genome Res. 14, 2469-2477. doi: 10.1101/gr.3024704

Chen, W.-M., de Faria, S. M., Chou, J.-H., James, E. K., Elliott, G. N., Sprent, J. I., et al. (2008). Burkholderia sabiae sp. nov., isolated from root nodules of Mimosa caesalpiniifolia. Int. J. Syst. Evol. Microbiol. 58, 2174-2179. doi: 10.1099/ijs.0.65816-0

Chen, W.-M., De Faria, S. M., James, E. K., Elliott, G. N., Lin, K.-Y., Chou, J.-H., et al. (2007). Burkholderia nodosa sp. nov., isolated from root nodules of the woody Brazilian legumes Mimosa bimucronata and Mimosa scabrella. Int. J. Syst. Evol. Microbiol. 57, 1055-1059. doi: 10.1099/ijs.0.64873-0

Chen, W.-M., James, E. K., Coenye, T., Chou, J.-H., Barrios, E., De Faria, S. M., et al. (2006). Burkholderia mimosarum sp. nov., isolated from root nodules of Mimosa spp. from Taiwan and South America. Int. J. Syst. Evol. Microbiol. 56, 1847-1851. doi: 10.1099/ijs.0.64325-0

Ciccarelli, F. D., Doerks, T., Von Mering, C., Creevey, C. J., Snel, B., and Bork, P. (2006). Toward automatic reconstruction of a highly resolved tree of life. Science 311, 1283-1287. doi: 10.1126/science.1123061

Coenye, T., Henry, D., Speert, D. P., and Vandamme, P. (2004). Burkholderia phenoliruptrix sp. nov., to accommodate the 2, 4, 5-trichlorophenoxyacetic acid and halophenol-degrading strain AC1100. Syst. Appl. Microbiol. 27, 623-627. doi: 10.1078/0723202042369992

Coenye, T., Laevens, S., Willems, A., Ohlén, M., Hannant, W., Govan, J., et al. (2001a). Burkholderia fungorum sp. nov. and Burkholderia caledonica sp. nov., two new species isolated from the environment, animals and human clinical samples. Int. J. Syst. Evol. Microbiol. 51, 1099-1107. doi: 10.1099/00207713-513-1099

Coenye, T., Mahenthiralingam, E., Henry, D., LiPuma, J. J., Laevens, S., Gillis, M., et al. (2001b). Burkholderia ambifaria sp. nov., a novel member of the Burkholderia cepacia complex including biocontrol and cystic fibrosis-related isolates. Int. J. Syst. Evol. Microbiol. 51, 1481-1490. doi: 10.1099/00207713-514-1481

Coenye, T., and Vandamme, P. (2003). Diversity and significance of Burkholderia species occupying diverse ecological niches. Environ. Microbiol. 5, 719-729. doi: 10.1046/j.1462-2920.2003.00471.x

Cole, J. R., Wang, Q., Cardenas, E., Fish, J., Chai, B., Farris, R. J., et al. (2009). The ribosomal database project: improved alignments and new tools for rRNA analysis. Nucleic Acids Res. 37, D141-D145. doi: 10.1093/nar/gkn879

Compant, S., Nowak, J., Coenye, T., Clement, C., and Ait Barka, E. (2008). Diversity and occurrence of Burkholderia spp. in the natural environment. FEMS Microbiol. Rev. 32, 607-626. doi: 10.1111/j.1574-6976.2008.00113.x 
Coutinho, B. G., Passos da Silva, D., Previato, J. O., Mendonca-Previato, L., and Venturi, V. (2013). Draft genome sequence of the rice endophyte Burkholderia kururiensis M130. Genome Announc. 1, e0022512-e022512. doi: 10.1128/genomeA.00225-12

De Meyer, S. E., Cnockaert, M., Ardley, J. K., Maker, G., Yates, R., Howieson, J. G., et al. (2013a). Burkholderia sprentiae sp. nov., isolated from Lebeckia ambigua root nodules. Int. J. Syst. Evol. Microbiol. 63(Pt 11), 3950-3957. doi: 10.1099/ijs.0.048777-0

De Meyer, S. E., Cnockaert, M., Ardley, J. K., Trengove, R. D., Garau, G., Howieson, J. G., et al. (2013b). Burkholderia rhynchosiae sp. nov., isolated from Rhynchosia ferulifolia root nodules. Int. J. Syst. Evol. Microbiol. 63(Pt 11), 3944-3949. doi: 10.1099/ijs.0.048751-0

De Meyer, S. E., Cnockaert, M., Ardley, J. K., Van Wyk, B.-E., Vandamme, P. A., and Howieson, J. G. (2014). Burkholderia dilworthii sp. nov., isolated from Lebeckia ambigua root nodules. Int. J. Syst. Evol. Microbiol. 64(Pt 4), 1090-1095. doi: 10.1099/ijs.0.058602-0

Deshazer, D. (2007). Virulence of clinical and environmental isolates of Burkholderia oklahomensis and Burkholderia thailandensis in hamsters and mice. FEMS Microbiol. Lett. 277, 64-69. doi: 10.1111/j.1574-6968.2007.00946.x

Estrada-de los Santos, P., Vinuesa, P., Martínez-Aguilar, L., Hirsch, A. M., and Caballero-Mellado, J. (2013). Phylogenetic analysis of Burkholderia species by multilocus sequence analysis. Curr. Microbiol. 67, 51-60. doi: 10.1007/s00284013-0330-9

Gao, B., and Gupta, R. S. (2012). Microbial systematics in the post-genomics era. Antonie Van Leeuwenhoek 101, 45-54. doi: 10.1007/s10482-011-9663-1

Gillis, M., Van Van, T., Bardin, R., Goor, M., Hebbar, P., Willems, A., et al. (1995). Polyphasic taxonomy in the genus Burkholderia leading to an emended description of the genus and proposition of Burkholderia vietnamiensis sp. nov. for N2-fixing isolates from rice in Vietnam. Int. J. Syst. Bacteriol. 45, 274-289. doi: 10.1099/00207713-45-2-274

Gogarten, J. P., Doolittle, W. F., and Lawrence, J. G. (2002). Prokaryotic evolution in light of gene transfer. Mol. Biol. Evol. 19, 2226-2238. doi: 10.1093/oxfordjournals.molbev.a004046

Goris, J., Dejonghe, W., Falsen, E., De Clerck, E., Geeraerts, B., Willems, A., et al. (2002). Diversity of transconjugants that acquired plasmid pJP4 or pEMT1 after inoculation of a donor strain in the A-and B-horizon of an agricultural soil and description of Burkholderia hospita sp. nov. and Burkholderia terricola sp. nov. Syst. Appl. Microbiol. 25, 340-352. doi: 10.1078/0723-2020-00134

Goris, J., De Vos, P., Caballero-Mellado, J., Park, J., Falsen, E., Quensen, J. F., et al. (2004). Classification of the biphenyl-and polychlorinated biphenyl-degrading strain LB400T and relatives as Burkholderia xenovorans sp. nov. Int. J. Syst. Evol. Microbiol. 54, 1677-1681. doi: 10.1099/ijs.0.63101-0

Gupta, R. S. (1998). Protein phylogenies and signature sequences: a reappraisal of evolutionary relationships among archaebacteria, eubacteria, and eukaryotes. Microbiol. Mol. Biol. Rev. 62, 1435.

Gupta, R. S. (2001). The branching order and phylogenetic placement of species from completed bacterial genomes, based on conserved indels found in various proteins. Int. Microbiol. 4, 187-202. doi: 10.1007/s10123-001-0037-9

Gupta, R. S. (2009). Protein signatures (molecular synapomorphies) that are distinctive characteristics of the major cyanobacterial clades. Int. J. Syst. Evol. Microbiol. 59, 2510. doi: 10.1099/ijs.0.005678-0

Gupta, R. S. (2014). Identification of Conserved Indels that are Useful for Classification and Evolutionary Studies Methods in Microbiology, Vol. 41. Oxford: Academic Press.

Gupta, R. S., and Griffiths, E. (2002). Critical issues in bacterial phylogeny. Theor. Popul. Biol. 61, 423-434. doi: 10.1006/tpbi.2002.1589

Gyaneshwar, P., Hirsch, A. M., Moulin, L., Chen, W.-M., Elliott, G. N., Bontemps, C., et al. (2011). Legume-nodulating betaproteobacteria: diversity, host range, and future prospects. Mol. Plant Microbe Interact. 24, 1276-1288. doi: 10.1094/MPMI-06-11-0172

Harris, J. K., Kelley, S. T., Spiegelman, G. B., and Pace, N. R. (2003). The genetic core of the universal ancestor. Genome Res. 13, 407-412. doi: 10.1101/gr.652803

Hauser, A. R., Jain, M., Bar-Meir, M., and McColley, S. A. (2011). Clinical significance of microbial infection and adaptation in cystic fibrosis. Clin. Microbiol. Rev. 24, 29-70. doi: 10.1128/CMR.00036-10

Holden, M. T., Seth-Smith, H. M., Crossman, L. C., Sebaihia, M., Bentley, S. D., Cerdeno-Tarraga, A. M., et al. (2009). The genome of Burkholderia cenocepacia J2315, an epidemic pathogen of cystic fibrosis patients. J. Bacteriol. 191, 261-277. doi: 10.1128/JB.01230-08
Holden, M. T., Titball, R. W., Peacock, S. J., Cerdeno-Tarraga, A. M., Atkins, T., Crossman, L. C., et al. (2004). Genomic plasticity of the causative agent of melioidosis, Burkholderia pseudomallei. Proc. Natl. Acad. Sci. U.S.A. 101, 14240-14245. doi: 10.1073/pnas.0403302101

Hong, K. W., Koh, C. L., Sam, C. K., Yin, W. F., and Chan, K. G. (2012) Complete genome sequence of Burkholderia sp. Strain GG4, a betaproteobacterium that reduces 3 -oxo- $\mathrm{N}$-acylhomoserine lactones and produces different N-acylhomoserine lactones. J. Bacteriol. 194, 6317-6312. doi: 10.1128/JB. 01578-12

Jeanmougin, F., Thompson, J. D., Gouy, M., Higgins, D. G., and Gibson, T. J. (1998). Multiple sequence alignment with Clustal X. Trends Biochem. Sci. 23, 403. doi: 10.1016/S0968-0004(98)01285-7

Jones, D. T., Taylor, W. R., and Thornton, J. M. (1992). The rapid generation of mutation data matrices from protein sequences. Comput. Appl. Biosci. CABIOS, 8, 275-282.

Kang, S. R., Srinivasan, S., and Lee, S. S. (2014). Burkholderia eburnea sp. nov. isolated from peat soil. Int. J. Syst. Evol. Microbiol. 64(Pt 4), 1108-1115. doi: 10.1099/ijs.0.051078-0

Katoh, K., and Standley, D. M. (2013). MAFFT multiple sequence alignment software version 7: improvements in performance and usability. Mol. Biol. Evol. 30, 772-780. doi: 10.1093/molbev/mst010

Khan, A., Asif, H., Studholme, D. J., Khan, I. A., and Azim, M. K. (2013). Genome characterization of a novel Burkholderia cepacia complex genomovar isolated from dieback affected mango orchards. World J. Microbiol. Biotechnol. 29, 2033-2044. doi: 10.1007/s11274-013-1366-5

Kim, H.-B., Park, M.-J., Yang, H.-C., An, D.-S., Jin, H.-Z., and Yang, D.-C. (2006) Burkholderia ginsengisoli sp. nov., a $\beta$-glucosidase-producing bacterium isolated from soil of a ginseng field. Int. J. Syst. Evol. Microbiol. 56, 2529-2533. doi: 10.1099/ijs.0.64387-0

Kim, H. S., Schell, M. A., Yu, Y., Ulrich, R. L., Sarria, S. H., Nierman, W. C., et al. (2005). Bacterial genome adaptation to niches: divergence of the potential virulence genes in three Burkholderia species of different survival strategies. BMC Genomics 6:174. doi: 10.1186/1471-2164-6-174

Kumar, S., Vikram, S., and Raghava, G. P. (2012). Genome sequence of the nitroaromatic compound-degrading Bacterium Burkholderia sp. strain SJ98. J. Bacteriol. 194, 3286-3212. doi: 10.1128/JB.00497-12

Kwak, M. J., Song, J. Y., Kim, S. Y., Jeong, H., Kang, S. G., Kim, B. K., et al. (2012). Complete genome sequence of the endophytic bacterium Burkholderia sp. strain KJ006. J. Bacteriol. 194, 4432-4433. doi: 10.1128/JB.00821-12

Kyrpides, N., Overbeek, R., and Ouzounis, C. (1999). Universal protein families and the functional content of the last universal common ancestor. J. Mol. Evol. 49, 413-423. doi: 10.1007/PL00006564

Lackner, G., Moebius, N., Partida-Martinez, L., and Hertweck, C. (2011). Complete genome sequence of Burkholderia rhizoxinica, an Endosymbiont of Rhizopus microsporus. J. Bacteriol. 193, 783-784. doi: 10.1128/JB.01318-10

Lee, C.-M., Weon, H.-Y., Yoon, S.-H., Kim, S.-J., Koo, B.-S., and Kwon, S.W. (2012). Burkholderia denitrificans sp. nov., isolated from the soil of Dokdo Island, Korea. J. Microbiol. 50, 855-859. doi: 10.1007/s12275-0121554-2

Lemaire, B., Robbrecht, E., van Wyk, B., Van Oevelen, S., Verstraete, B., Prinsen, E., et al. (2011). Identification, origin, and evolution of leaf nodulating symbionts of Sericanthe (Rubiaceae). J. Microbiol. 49, 935-941. doi: 10.1007/s12275-0111163-5

Lemaire, B., Van Oevelen, S., De Block, P., Verstraete, B., Smets, E., Prinsen, E., et al. (2012). Identification of the bacterial endosymbionts in leaf nodules of Pavetta (Rubiaceae). Int. J. Syst. Evol. Microbiol. 62, 202-209. doi: 10.1099/ijs.0.02 8019-0

Lim, J. H., Baek, S.-H., and Lee, S.-T. (2008). Burkholderia sediminicola sp. nov. isolated from freshwater sediment. Int. J. Syst. Evol. Microbiol. 58, 565-569. doi: 10.1099/ijs.0.65502-0

Lim, J., Lee, T. H., Nahm, B. H., Choi, Y. D., Kim, M., and Hwang, I. (2009). Complete genome sequence of Burkholderia glumae BGR1. J. Bacteriol. 191, 3758-3759. doi: 10.1128/JB.00349-09

Lim, J. S., Choi, B. S., Choi, A. Y., Kim, K. D., Kim, D. I., Choi, I. Y., et al. (2012). Complete genome sequence of the fenitrothion-degrading Burkholderia sp. strain YI23. J. Bacteriol. 194, 896-811. doi: 10.1128/JB.06479-11

Lim, Y. W., Baik, K. S., Han, S. K., Kim, S. B., and Bae, K. S. (2003). Burkholderia sordidicola sp. nov., isolated from the white-rot fungus Phanerochaete sordida. Int. J. Syst. Evol. Microbiol. 53, 1631-1636. doi: 10.1099/ijs.0.02456-0 
Limmathurotsakul, D., and Peacock, S. J. (2011). Melioidosis: a clinical overview. Br. Med. Bull. 99, 125-139. doi: 10.1093/bmb/ldr007

Lu, P., Zheng, L.-Q., Sun, J.-J., Liu, H.-M., Li, S.-P., Hong, Q., et al. (2012). Burkholderia zhejiangensis sp. nov., a methyl-parathion-degrading bacterium isolated from a wastewater-treatment system. Int. J. Syst. Evol. Microbiol. $62(\mathrm{Pt}$ 6), 1337-1341. doi: 10.1099/ijs.0.035428-0

Mahenthiralingam, E., Baldwin, A., and Vandamme, P. (2002). Burkholderia cepacia complex infection in patients with cystic fibrosis. J. Med. Microbiol. 51, 533-538.

Mahenthiralingam, E., Urban, T. A., and Goldberg, J. B. (2005). The multifarious, multireplicon Burkholderia cepacia complex. Nat. Rev. Microbiol. 3, 144-156. doi: $10.1038 /$ nrmicro1085

Mardis, E. R. (2008). The impact of next-generation sequencing technology on genetics. Trends Genet. 24, 133-141. doi: 10.1016/j.tig.2007.12.007

Martínez-Aguilar, L., Salazar-Salazar, C., Méndez, R. D., Caballero-Mellado, J., Hirsch, A. M., Vásquez-Murrieta, M. S., et al. (2013). Burkholderia caballeronis sp. nov., a nitrogen fixing species isolated from tomato (Lycopersicon esculentum) with the ability to effectively nodulate Phaseolus vulgaris. Antonie Van Leeuwenhoek 104, 1063-1071. doi: 10.1007/s10482-013-0028-9

Mavengere, N. R., Ellis, A. G., and Le Roux, J. J. (2014). Burkholderia aspalath sp. nov., isolated from root nodules of the South African legume Aspalathus abietina Thunb. Int. J. Syst. Evol. Microbiol. 64, 1906-1912. doi: 10.1099/ijs.0. 057067-0

Nandakumar, R., Shahjahan, A., Yuan, X., Dickstein, E., Groth, D., Clark, C., et al. (2009). Burkholderia glumae and B. gladioli cause bacterial panicle blight in rice in the southern United States. Plant Dis. 93, 896-905. doi: 10.1094/PDIS-93-90896

Nazir, R., Hansen, M. A., Sorensen, S., and van Elsas, J. D. (2012). Draft genome sequence of the soil bacterium Burkholderia terrae strain BS001, which interacts with fungal surface structures. J. Bacteriol. 194, 4480-4481. doi: 10.1128/J. B. $00725-12$

NCBI. (2014). NCBI Genome Database. http://www.ncbi.nlm.nih.gov/genome/

Nierman, W. C., DeShazer, D., Kim, H. S., Tettelin, H., Nelson, K. E., Feldblyum, T., et al. (2004). Structural flexibility in the Burkholderia mallei genome. Proc. Natl. Acad. Sci. U.S.A. 101, 14246-14251. doi: 10.1073/pnas.0403 306101

O'Carroll, M. R., Kidd, T. J., Coulter, C., Smith, H. V., Rose, B. R., Harbour, C., et al. (2003). Burkholderia pseudomallei: another emerging pathogen in cystic fibrosis. Thorax 58, 1087-1091. doi: 10.1136/thorax.58.12.1087

Oliveira Cunha, C., Goda Zuleta, L. F., Paula de Almeida, L. G., Prioli Ciapina, L., Lustrino Borges, W., Pitard, R. M., et al. (2012). Complete genome sequence of Burkholderia phenoliruptrix BR3459a (CLA1), a heat-tolerant, nitrogen-fixing symbiont of Mimosa flocculosa. J. Bacteriol. 194, 6675-6676. doi: 10.1128/JB.01821-12

Onofre-Lemus, J., Hernández-Lucas, I., Girard, L., and Caballero-Mellado, J. (2009). ACC (1-aminocyclopropane-1-carboxylate) deaminase activity, a widespread trait in Burkholderia species, and its growth-promoting effect on tomato plants. Appl. Environ. Microbiol. 75, 6581-6590. doi: 10.1128/AEM. 01240-09

Ormeno-Orrillo, E., Rogel, M. A., Chueire, L. M., Tiedje, J. M., Martinez-Romero, E., and Hungria, M. (2012). Genome sequences of Burkholderia sp. strains CCGE1002 and H160, isolated from legume nodules in Mexico and Brazil. J. Bacteriol. 194, 6927-6912. doi: 10.1128/JB.01756-12

Otsuka, Y., Muramatsu, Y., Nakagawa, Y., Matsuda, M., Nakamura, M., and Murata, H. (2011). Burkholderia oxyphila sp. nov., a bacterium isolated from acidic forest soil that catabolizes (+)-catechin and its putative aromatic derivatives. Int. J. Syst. Evol. Microbiol. 61, 249-254. doi: 10.1099/ijs.0.017368-0

Palleroni, N. J. (2005). "Genus I. Burkholderia Yabuuchi et al. 1993, $398^{V P}$ (Effective publication: Yabuuchi et al. 1992, 1268) emend. Gillis et al. 1995, 286*” in Bergey's Manual of Systematic Bacteriology, 2 Edn, Vol. 2, eds D. J. Brenner, N. R. Krieg, G. M. Garrity, and J. T. Staley (New York, NY: Springer), 575-600.

Parte, A. C. (2013). LPSN-list of prokaryotic names with standing in nomenclature. Nucleic Acids Res. 42, D613-D616. doi: 10.1093/nar/gkt1111

Partida-Martinez, L. P., Groth, I., Schmitt, I., Richter, W., Roth, M., and Hertweck, C. (2007). Burkholderia rhizoxinica sp. nov. and Burkholderia endofungorum sp. nov., bacterial endosymbionts of the plant-pathogenic fungus Rhizopus microsporus. Int. J. Syst. Evol. Microbiol. 57, 2583-2590. doi: 10.1099/ijs.0. 64660-0

Payne, G. W., Vandamme, P., Morgan, S. H., LiPuma, J. J., Coenye, T., Weightman, A. J., et al. (2005). Development of a recA gene-based identification approach for the entire Burkholderia genus. Appl. Environ. Microbiol. 71, 3917-3927. doi: 10.1128/AEM.71.7.3917-3927.2005

Perin, L., Martínez-Aguilar, L., Paredes-Valdez, G., Baldani, J., Estrada-de Los Santos, P., Reis, V., et al. (2006). Burkholderia silvatlantica sp. nov., a diazotrophic bacterium associated with sugar cane and maize. Int. J. Syst. Evol. Microbiol. 56, 1931-1937. doi: 10.1099/ijs.0.64362-0

Reis, V., Estrada-De los Santos, P., Tenorio-Salgado, S., Vogel, J., Stoffels, M., Guyon, S., et al. (2004). Burkholderia tropica sp. nov., a novel nitrogen-fixing, plant-associated bacterium. Int. J. Syst. Evol. Microbiol. 54, 2155-2162. doi: 10.1099/ijs.0.02879-0

Rokas, A., and Holland, P. W. H. (2000). Rare genomic changes as a tool for phylogenetics. Trends Ecol. Evol. 15, 454-459. doi: 10.1016/S0169-5347(00)01967-4

Segata, N., Bornigen, D., Morgan, X. C., and Huttenhower, C. (2013). PhyloPhlAn is a new method for improved phylogenetic and taxonomic placement of microbes. Nat. Commun. 4, 2304. doi: 10.1038/ncomms3304

Seo, Y. S., Lim, J., Choi, B. S., Kim, H., Goo, E., Lee, B., et al. (2011). Complete genome sequence of Burkholderia gladioli BSR3. J. Bacteriol. 193, 3149-3111. doi: 10.1128/JB.00420-11

Sessitsch, A., Coenye, T., Sturz, A., Vandamme, P., Barka, E. A., Salles, J., et al. (2005). Burkholderia phytofirmans sp. nov., a novel plant-associated bacterium with plant-beneficial properties. Int. J. Syst. Evol. Microbiol. 55, 1187-1192. doi: 10.1099/ijs.0.63149-0

Sheu, S.-Y., Chou, J.-H., Bontemps, C., Elliott, G. N., Gross, E., James, E. K., et al. (2012). Burkholderia symbiotica sp. nov., isolated from root nodules of Mimosa spp. native to north-east Brazil. Int. J. Syst. Evol. Microbiol. 62(Pt 9), 2272-2278. doi: 10.1099/ijs.0.037408-0

Sheu, S.-Y., Chou, J.-H., Bontemps, C., Elliott, G. N., Gross, E., dos Reis Junior, F. B., et al. (2013). Burkholderia diazotrophica sp. nov., isolated from root nodules of Mimosa spp. Int. J. Syst. Evol. Microbiol. 63(Pt 2), 435-441. doi: 10.1099/ijs.0.039859-0

Shibata, T. F., Maeda, T., Nikoh, N., Yamaguchi, K., Oshima, K., Hattori, M., et al. (2013). Complete genome sequence of Burkholderia sp. Strain RPE64, bacterial symbiont of the bean bug Riptortus pedestris. Genome Announc. 1, 10-13. doi: 10.1128/genomeA.00441-13

Simpson, I. N., Finlay, J., Winstanley, D. J., Dewhurst, N., Nelson, J. W., Butler, S. L., et al. (1994). Multi-resistance isolates possessing characteristics of both Burkholderia (Pseudomonas) cepacia and Burkholderia gladiol from patients with cystic fibrosis. J. Antimicrob. Chemother. 34, 353-361. doi: 10.1093/jac/34.3.353

Song, J. Y., Kwak, M. J., Lee, K. Y., Kong, H. G., Kim, B. K., Kwon, S. K., et al. (2012). Draft genome sequence of the antifungal-producing plant-benefiting bacterium Burkholderia pyrrocinia CH-67. J. Bacteriol. 194, 6649-6650. doi: 10.1128/JB.01779-12

Spilker, T., Baldwin, A., Bumford, A., Dowson, C. G., Mahenthiralingam, E., and LiPuma, J. J. (2009). Expanded multilocus sequence typing for Burkholderia species. J. Clin. Microbiol. 47, 2607-2610. doi: 10.1128/JCM.00770-09

Stead, D. (1992). Grouping of plant-pathogenic and some other Pseudomonas spp. by using cellular fatty acid profiles. Int. J. Syst. Bacteriol. 42, 281-295. doi: 10.1099/00207713-42-2-281

Suarez-Moreno, Z. R., Caballero-Mellado, J., Coutinho, B. G., Mendonca-Previato, L., James, E. K., and Venturi, V. (2012). Common features of environmental and potentially beneficial plant-associated Burkholderia. Microb. Ecol. 63, 249-266. doi: 10.1007/s00248-011-9929-1

Tamura, K., Stecher, G., Peterson, D., Filipski, A., and Kumar, S. (2013). MEGA6: Molecular Evolutionary Genetics Analysis version 6.0. Mol. Biol. Evol. 30, 2725-2729. doi: 10.1093/molbev/mst197

Tavaré, S. (1986). "Some probabilistic and statistical problems in the analysis of DNA sequences," in Lectures on Mathematics in the Life Sciences, 17 Edn, ed R. M. Miura (Providence (RI): American Mathematical Society), 57-86.

Tayeb, L. A., Lefevre, M., Passet, V., Diancourt, L., Brisse, S., and Grimont, P. A. (2008). Comparative phylogenies of Burkholderia, Ralstonia, Comamonas, Brevundimonas and related organisms derived from $r p o B, g y r B$ and $r r s$ gene sequences. Res. Microbiol. 159, 169-177. doi: 10.1016/j.resmic.2007.12.005

Tian, Y., Kong, B. H., Liu, S. L., Li, C. L., Yu, R., Liu, L., et al. (2013). Burkholderia grimmiae sp. nov., isolated from a xerophilous moss (Grimmia montana). Int. J. Syst. Evol. Microbiol. 63(Pt 6), 2108-2113. doi: 10.1099/ijs.0.045492-0

Ussery, D. W., Kiil, K., Lagesen, K., Sicheritz-Ponten, T., Bohlin, J., and Wassenaar, T. M. (2009). The genus Burkholderia: analysis of 56 genomic sequences. Genome Dyn. 6, 140-457. doi: 10.1159/000235768 
Valverde, A., Delvasto, P., Peix, A., Velázquez, E., Santa-Regina, I., Ballester, A., et al. (2006). Burkholderia ferrariae sp. nov., isolated from an iron ore in Brazil. Int. J. Syst. Evol. Microbiol. 56, 2421-2425. doi: 10.1099/ijs.0.64498-0

Vandamme, P., and Dawyndt, P. (2011). Classification and identification of the Burkholderia cepacia complex: past, present and future. Syst. Appl. Microbiol. 34, 87-95. doi: 10.1016/j.syapm.2010.10.002

Vandamme, P., De Brandt, E., Houf, K., Salles, J. F., van Elsas, J. D., Spilker, T., et al. (2013). Burkholderia humi sp. nov., Burkholderia choica sp. nov., Burkholderia telluris sp. nov., Burkholderia terrestris sp. nov. and Burkholderia udeis sp. nov.: Burkholderia glathei-like bacteria from soil and rhizosphere soil. Int. J. Syst. Evol. Microbiol. 63(Pt 12), 4707-4718. doi: 10.1099/ijs.0.048900-0

Vandamme, P., Goris, J., Chen, W. M., de Vos, P., and Willems, A. (2002b). Burkholderia tuberum sp. nov. and Burkholderia phymatum sp. nov., nodulate the roots of tropical legumes. Syst. Appl. Microbiol. 25, 507-512. doi: $10.1078 / 07232020260517634$

Vandamme, P., Goris, J., Chen, W.-M., De Vos, P., and Willems, A. (2002a). Burkholderia tuberum sp. nov. and Burkholderia phymatum sp. nov., nodulate the roots of tropical legumes. Syst. Appl. Microbiol. 25, 507-512. doi $10.1078 / 07232020260517634$

Vandamme, P., Holmes, B., Vancanneyt, M., Coenye, T., Hoste, B., Coopman, R., et al. (1997). Occurrence of multiple genomovars of Burkholderia cepacia in cystic fibrosis patients and proposal of Burkholderia multivorans sp. nov. Int. J. Syst. Bacteriol. 47, 1188-1200. doi: 10.1099/00207713-47-4-1188

Vandamme, P., Opelt, K., Knöchel, N., Berg, C., Schönmann, S., De Brandt, E., et al. (2007). Burkholderia bryophila sp. nov. and Burkholderia megapolitana sp. nov., moss-associated species with antifungal and plant-growth-promoting properties. Int. J. Syst. Evol. Microbiol. 57, 2228-2235. doi: 10.1099/ijs.0.65142-0

Vanlaere, E., van der Meer, J. R., Falsen, E., Salles, J. F., De Brandt, E., and Vandamme, P. (2008). Burkholderia sartisoli sp. nov., isolated from a polycyclic aromatic hydrocarbon-contaminated soil. Int. J. Syst. Evol. Microbiol. 58, 420-423. doi: 10.1099/ijs.0.65451-0

Van Oevelen, S., De Wachter, R., Vandamme, P., Robbrecht, E., and Prinsen, E. (2002a). Identification of the bacterial endosymbionts in leaf galls of Psychotria (Rubiaceae, angiosperms) and proposal of'Candidatus Burkholderia kirkii'sp. nov. Int. J. Syst. Evol. Microbiol. 52, 2023-2027. doi: 10.1099/ijs.0.02103-0

Van Oevelen, S., De Wachter, R., Vandamme, P., Robbrecht, E., and Prinsen, E. (2002b). Identification of the bacterial endosymbionts in leaf galls of Psychotria (Rubiaceae, angiosperms) and proposal of 'Candidatus Burkholderia kirkii' sp. nov. Int. J. Syst. Evol. Microbiol. 52(Pt 6), 2023-2027. doi: 10.1099/ijs.0. 02103-0

Van Oevelen, S., De Wachter, R., Vandamme, P., Robbrecht, E., and Prinsen, E. (2004). 'Candidatus Burkholderia calva' and 'Candidatus Burkholderia nigropunctata' as leaf gall endosymbionts of African Psychotria. Int. J. Syst. Evol. Microbiol. 54, 2237-2239. doi: 10.1099/ijs.0.63188-0

Viallard, V., Poirier, I., Cournoyer, B., Haurat, J., Wiebkin, S., Ophel-Keller, K., et al. (1998). Burkholderia graminis sp. nov., a rhizospheric Burkholderia species, and reassessment of [Pseudomonas] phenazinium,[Pseudomonas] pyrrocinia and [Pseudomonas] glathei as Burkholderia. Int. J. Syst. Bacteriol. 48, 549-563. doi: 10.1099/00207713-48-2-549

Weilharter, A., Mitter, B., Shin, M. V., Chain, P. S., Nowak, J., and Sessitsch, A. (2011). Complete genome sequence of the plant growth-promoting endophyte Burkholderia phytofirmans strain PsJN. J. Bacteriol. 193, 3383-3384. doi 10.1128/JB.05055-11

White, N. J. (2003). Melioidosis. Lancet 361, 1715. doi: 10.1016/S01406736(03)13374-0

Whitlock, G. C., Estes, D. M., and Torres, A. G. (2007). Glanders: off to the races with Burkholderia mallei. FEMS Microbiol. Lett. 277, 115-122. doi: 10.1111/j.1574-6968.2007.00949.x
Wiersinga, W. J., van der Poll, T., White, N. J., Day, N. P., and Peacock, S. J. (2006). Melioidosis: insights into the pathogenicity of Burkholderia pseudomallei. Nat. Rev. Microbiol. 4, 272-282. doi: 10.1038/nrmicro1385

Wong, S. Y., Paschos, A., Gupta, R. S., and Schellhorn, H. E. (2014). Insertion/deletion-based approach for the detection of Escherichia coli O157:H7 in freshwater environments. Environ. Sci. Technol. 48, 11462-11470. doi: $10.1021 /$ es502794h

Wu, D., Hugenholtz, P., Mavromatis, K., Pukall, R., Dalin, E., Ivanova, N. N., et al. (2009). A phylogeny-driven genomic encyclopaedia of Bacteria and Archaea. Nature 462, 1056-1060. doi: 10.1038/nature08656

Yabuuchi, E., Kosako, Y., Oyaizu, H., Yano, I., Hotta, H., Hashimoto, Y., et al. (1992). Proposal of Burkholderia gen. nov. and transfer of seven species of the genus Pseudomonas homology group II to the new genus, with the type species Burkholderia cepacia (Palleroni and Holmes 1981) comb. nov. Microbiol. Immunol. 36, 1251-1275. doi: 10.1111/j.1348-0421.1992.tb02129.x

Yabuuchi, E., Kosako, Y., Oyaizu, H., Yano, I., Hotta, H., Hashimoto, Y., et al. (1993). Burkholderia gen. nov. validation of the publication of new names and new combinations previously effectively published outside the IJSB, List no 43. Int. J. Syst. Bacteriol. 43, 398-399. doi: 10.1099/00207713-43-2-398

Yang, H.-C., Im, W.-T., Kim, K. K., An, D.-S., and Lee, S.-T. (2006). Burkholderia terrae sp. nov., isolated from a forest soil. Int. J. Syst. Evol. Microbiol. 56, 453-457. doi: 10.1099/ijs.0.63968-0

Yarza, P., Richter, M., Peplies, J., Euzeby, J., Amann, R., Schleifer, K. H., et al. (2008). The All-Species Living Tree project: A 16S rRNA-based phylogenetic tree of all sequenced type strains. Syst. Appl. Microbiol. 31, 241-250. doi: 10.1016/j.syapm.2008.07.001

Yoo, S.-H., Kim, B.-Y., Weon, H.-Y., Kwon, S.-W., Go, S.-J., and Stackebrandt, E. (2007). Burkholderia soli sp. nov., isolated from soil cultivated with Korean ginseng. Int. J. Syst. Evol. Microbiol. 57, 122-125. doi: 10.1099/ijs.0. 64471-0

Zhang, H., Hanada, S., Shigematsu, T., Shibuya, K., Kamagata, Y., Kanagawa, T., et al. (2000). Burkholderia kururiensis sp. nov., a trichloroethylene (TCE)-degrading bacterium isolated from an aquifer polluted with TCE. Int. J. Syst. Evol. Microbiol. 50, 743-749. doi: 10.1099/0020771350-2-743

Zhu, B., Zhou, S., Lou, M., Zhu, J., Li, B., Xie, G., et al. (2011). Characterization and inference of gene gain/loss along burkholderia evolutionary history. Evol. Bioinform. Online 7, 191. doi: 10.4137/EBO.S7510

Conflict of Interest Statement: The authors declare that the research was conducted in the absence of any commercial or financial relationships that could be construed as a potential conflict of interest.

Received: 18 September 2014; paper pending published: 24 October 2014; accepted: 21 November 2014; published online: 19 December 2014.

Citation: Sawana A, Adeolu M and Gupta RS (2014) Molecular signatures and phylogenomic analysis of the genus Burkholderia: proposal for division of this genus into the emended genus Burkholderia containing pathogenic organisms and a new genus Paraburkholderia gen. nov. harboring environmental species. Front. Genet. 5:429. doi: 10.3389/fgene.2014.00429

This article was submitted to Evolutionary and Genomic Microbiology, a section of the journal Frontiers in Genetics.

Copyright (c) 2014 Sawana, Adeolu and Gupta. This is an open-access article distributed under the terms of the Creative Commons Attribution License (CC BY). The use, distribution or reproduction in other forums is permitted, provided the original author(s) or licensor are credited and that the original publication in this journal is cited, in accordance with accepted academic practice. No use, distribution or reproduction is permitted which does not comply with these terms. 